\title{
Three-dimensional Navier-Stokes simulations of buoyant, vertical miscible Hele-Shaw displacements
}

\section{Journal Article}

Author(s):

Heussler, F.H.C.; Oliveira, Rafael; John, Michael O.; Meiburg, Eckart H.

Publication date:

2014-08-10

Permanent link:

https://doi.org/10.3929/ethz-b-000086484

Rights / license:

In Copyright - Non-Commercial Use Permitted

Originally published in:

Journal of Fluid Mechanics 752, https://doi.org/10.1017/jfm.2014.327 


\title{
Three-dimensional Navier-Stokes simulations of buoyant, vertical miscible Hele-Shaw displacements
}

\author{
F. H. C. Heussler ${ }^{1,2}$, R. M. Oliveira ${ }^{2, \dagger}$, M. O. John ${ }^{2,3}$ and E. Meiburg ${ }^{2, \dagger}$ \\ ${ }^{1}$ Rheinisch-Westfaelische Technische Hochschule Aachen, D-52056 Aachen, Germany \\ ${ }^{2}$ Department of Mechanical Engineering, University of California at Santa Barbara, Santa Barbara, \\ CA 93106, USA \\ ${ }^{3}$ ETH, Institute of Fluid Dynamics, CH-8092 Zurich, Switzerland
}

(Received 23 November 2013; revised 30 April 2014; accepted 4 June 2014; first published online 2 July 2014)

\begin{abstract}
Gravitationally and viscously unstable miscible displacements in vertical Hele-Shaw cells are investigated via three-dimensional Navier-Stokes simulations. The velocity of the two-dimensional base-flow displacement fronts generally increases with the unfavourable viscosity contrast and the destabilizing density difference. Displacement fronts moving faster than the maximum velocity of the Poiseuille flow far downstream exhibit a single stagnation point in a moving reference frame, consistent with earlier observations for corresponding capillary tube flows. Gravitationally stable fronts, on the other hand, can move more slowly than the Poiseuille flow, resulting in more complex streamline patterns and the formation of a spike at the tip of the front, in line with earlier findings. A two-dimensional pinch-off governed by dispersion is observed some distance behind the displacement front. Three-dimensional simulations of viscously and gravitationally unstable vertical displacements show a strong vorticity quadrupole along the length of the finger, similar to recent observations for neutrally buoyant flows. This quadrupole results in an inner splitting instability of vertically propagating fingers. Even though the quadrupole's strength increases for larger destabilizing density differences, the inner splitting is delayed due to the presence of a secondary, outer quadrupole which counteracts the inner one. For large unstable density differences, the formation of a secondary, downward-propagating front is observed, which is also characterized by inner and outer vorticity quadrupoles. This front develops an anchor-like shape as a result of the flow induced by these quadrupoles. Increased spanwise wavelengths of the initial perturbation are seen to result in the formation of the well-known tip-splitting instability. For suitable initial conditions, the inner and tip-splitting instabilities can be seen to develop side by side, affecting different regions of the flow field.
\end{abstract}

Key words: fingering instability, Hele-Shaw flows, low-Reynolds-number flows

$\dagger$ Email address for correspondence: meiburg@engineering.ucsb.edu $\ddagger$ Present address: Halliburton Brazil Technology Center, Halliburton, Rio de Janeiro, RJ 21941-907, Brazil. 


\section{Introduction}

Displacements in Hele-Shaw cells, popular as models for corresponding porous media flows, have revealed a wealth of information about viscously and gravitationally driven instabilities (see, e.g., Homsy 1987). In particular, they have provided fundamental insight into the viscous fingering phenomenon and associated issues of pattern formation (see, e.g., Maxworthy 1987). Efforts to simulate such flows computationally have generally employed two-dimensional gap-averaged equations such as Darcy's law and variations thereof. This approach has been successful in reproducing many of the experimentally observed phenomena, such as the evolution of pronounced fingers, as well as their tip-splitting and merging behaviour (e.g. Tan \& Homsy 1988; Chen \& Meiburg 1998; Ruith \& Meiburg 2000). Nevertheless, at least for immiscible displacements, it was established long ago that three-dimensional effects become important in the vicinity of the moving interface (Park \& Homsy 1984). Recently, Oliveira \& Meiburg (2011) presented the first fully three-dimensional Navier-Stokes simulations of neutrally buoyant, miscible Hele-Shaw displacements. These simulations revealed some surprising flow features, such as the presence of strong streamwise vorticity quadrupoles along the length of the finger. These promote a previously unidentified 'inner splitting' instability of the fingers by transporting resident, more-viscous fluid from the walls of the cell towards the centre of the finger, while convecting injected, less-viscous fluid laterally away from the centre of the finger in the spanwise direction. The question then arises as to how these streamwise vorticity structures will be modified by gravitational forces, if the two fluids have different densities. For miscible displacements in horizontal Hele-Shaw cells, this issue has been addressed in the recent linear stability work of Talon, Goyal \& Meiburg (2013) and the corresponding nonlinear simulations by John et al. (2013). Talon et al. (2013) found that two-dimensional base states allow for two distinct spanwise instability modes; one is a tip, viscously driven instability, and the other is a gravitational instability that occurs at the unstably stratified horizontal interface along the side of the finger. Their linear stability analysis demonstrates that the dominant wavelength of the gravitational instability is generally smaller than that of the viscous instability. John et al. (2013) observed that the influence of gravity breaks the up/down symmetry of the flow and hence of the vorticity quadrupole. Instead, a vorticity dipole structure is favoured that is associated with a gravitational instability and which can also result in a longitudinal splitting of the finger.

By contrast, the symmetry properties of variable-density displacements in vertical Hele-Shaw cells are such that the existence of streamwise vorticity quadrupoles similar to those in the neutrally buoyant case appears possible. Whether or not such vorticity structures indeed exist in vertical displacements and how they might be modified by gravitational forces are the key questions to be addressed in the present investigation. This vertical miscible displacement configuration has been addressed experimentally in the past by Lajeunesse et al. (1997, 1999, 2001), who provided information on the two-dimensional base state as well as on the stability threshold. These authors furthermore observed a dominant fingering wavelength of approximately five times the gap width, which agrees well with the linear stability data of Goyal, Pichler \& Meiburg (2007). In the current investigation, we carry out two-dimensional simulations to describe the evolution of the base state in gravitationally stable and unstable configurations. Subsequently, we will perform three-dimensional Navier-Stokes simulations of variable-density and variable-viscosity miscible displacements in vertical Hele-Shaw cells to analyse the influence of gravitational effects on vorticity quadrupoles and nonlinear pattern formation. The 


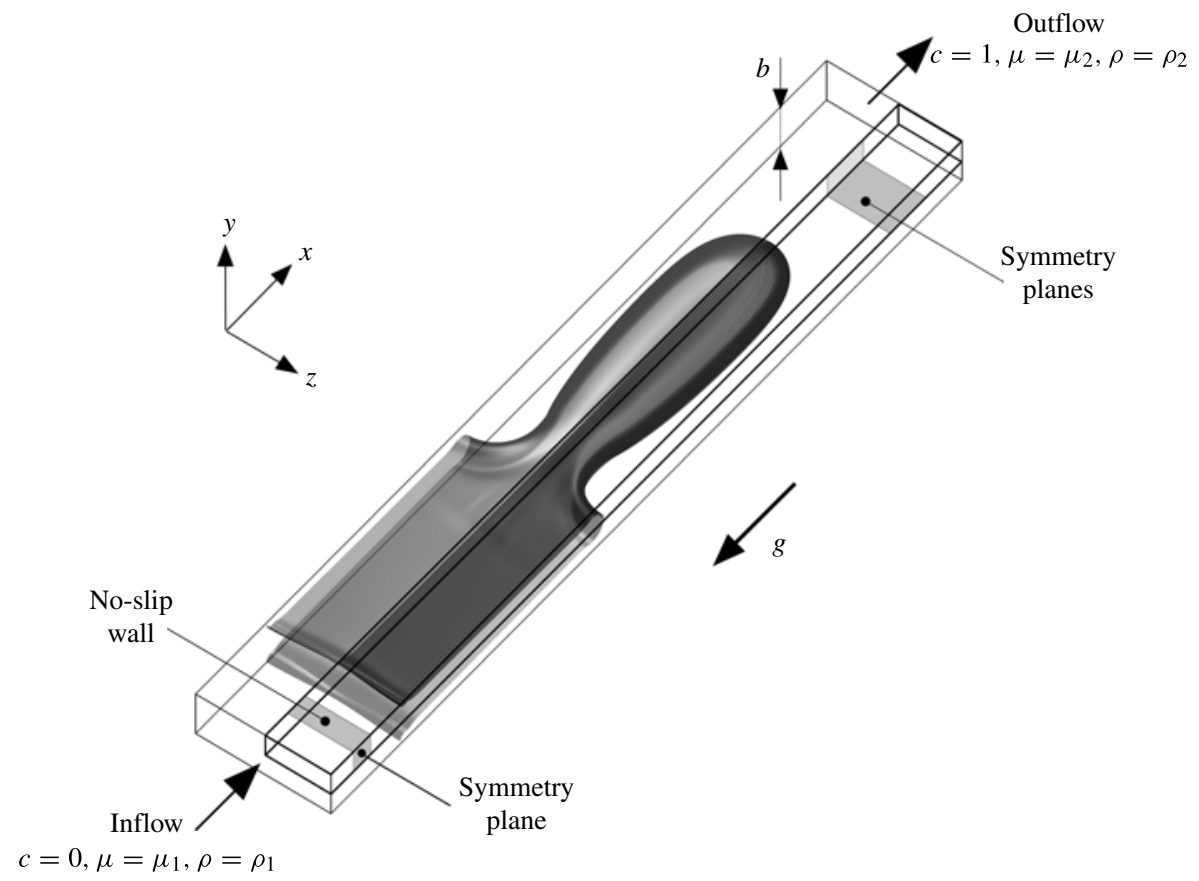

FIGURE 1. A less-viscous fluid with viscosity $\mu_{1}$ and density $\rho_{1}$ displaces a more-viscous resident fluid with viscosity $\mu_{2}$ and density $\rho_{2}$ in the upward direction, $+x$. Two vertical symmetry planes exist at $y=0$ and $z=0$, so that only the dark-grey section where $y>0$ and $z>0$ is simulated.

two-dimensional simulations reveal several possible configurations for the streamline patterns, from single stagnation points in gravitationally unstable displacements to multiple stagnation points and recirculation zones as well as spike formation in stable configurations. Some of these findings are consistent with previous observations for capillary tube flows by Chen \& Meiburg (1996), Petitjeans \& Maxworthy (1996) and Kuang, Petitjeans \& Maxworthy (2004); see also the results of Soares \& Thompson (2009) for immiscible displacements. The three-dimensional Navier-Stokes simulations show that the presence of strong streamwise vorticity quadrupoles leads to the formation of an inner splitting instability in the upward-propagating viscous finger, similar to the recent observations for neutrally buoyant flows by Oliveira \& Meiburg (2011). For large unstable density differences, the Rayleigh-Taylor instability is seen to result in a reverse flow that gives rise to an anchor-like structure.

The outline of the paper is as follows. In $\S 2$ the flow configuration and governing equations will be defined, and in $\S \S 3$ and 4 we describe the computational approach and present validation information, respectively. Section 5 focuses on the evolution and key properties of the two-dimensional base flow, and $\S 6$ discusses in detail the threedimensional flows that evolve upon perturbing the two-dimensional base flow with a spanwise wave. Section 7 presents the first three-dimensional simulations of the wellknown tip-splitting instability and its concurrent growth with the new inner splitting instability. Finally, $\S 8$ summarizes our findings.

\section{Physical problem}

We consider a vertical Hele-Shaw cell consisting of two parallel flat plates separated by a gap of constant width $b$; see figure 1 . The cell contains two miscible fluids 
of different viscosities $\mu$ and densities $\rho$. The less-viscous fluid of viscosity $\mu_{1}$ pushes the more-viscous fluid with viscosity $\mu_{2}$ in the upward, $+x$, direction, so that the displacement is viscously unstable. The density difference $\Delta \rho=\rho_{2}-\rho_{1}$ can be either stabilizing or destabilizing. The spatiotemporal evolution of the displacement is governed by the three-dimensional incompressible transient Navier-Stokes equations in the Boussinesq approximation, coupled to a convection-diffusion equation for the concentration field. In terms of non-dimensional variables, these equations read

$$
\begin{gathered}
\frac{\partial u_{k}}{\partial x_{k}}=0 \\
\frac{\partial u_{i}}{\partial t}+u_{k} \frac{\partial u_{i}}{\partial x_{k}}=\frac{1}{R e}\left[\frac{\partial}{\partial x_{k}} \mu\left(\frac{\partial u_{i}}{\partial x_{k}}+\frac{\partial u_{k}}{\partial x_{i}}\right)-\frac{\partial p}{\partial x_{i}}-\delta_{i 1} F c\right] \\
\frac{\partial c}{\partial t}+u_{k} \frac{\partial c}{\partial x_{k}}=\frac{1}{P e} \frac{\partial}{\partial x_{k}} \frac{\partial c}{\partial x_{k}}
\end{gathered}
$$

A Cartesian coordinate system $x_{i}=(x, y, z)$ is used, with $x$ specifying the streamwise, upward direction and $y$ and $z$ denoting the cross-gap and spanwise directions, respectively. The velocity components in these directions are indicated by $u_{i}$, while $c$ represents the relative concentration of the more-viscous fluid, $p$ denotes pressure and $t$ denotes time. Density and dynamic viscosity are assumed to be related to $c$ by

$$
\begin{gathered}
\rho=\rho_{1}+c\left(\rho_{2}-\rho_{1}\right)=\rho_{1}+c \Delta \rho, \\
\mu=\mu_{2} \mathrm{e}^{M(c-1)} .
\end{gathered}
$$

The viscosity contrast between the two fluids is defined as $M=\ln \left(\mu_{2} / \mu_{1}\right)$. Using the characteristic length $L=b$, time $T=b / U$ and pressure $P=\mu_{2} U / b$, where $U$ is the gapaveraged inflow velocity, the non-dimensional parameters are defined as follows. The Reynolds number $R e=\rho_{2} U b / \mu_{2}$ and the Péclet number $P e=U b / D$, where $D$ denotes a constant diffusion coefficient, represent the ratio of advective to diffusive transport in the momentum and species conservation equations, respectively. We will also make use of the alternative Reynolds number $R e^{*}=\rho_{1} U b / \mu_{1}=\operatorname{Re} \mathrm{e}^{M}$. Here $\operatorname{Re}$ will be more convenient for comparing our results with the linear stability results of Goyal et al. (2007) for validation purposes, but we will employ $R e^{*}$ when making comparisons with the neutrally buoyant nonlinear simulation results of Oliveira \& Meiburg (2011). The gravity number $F=\Delta \rho g b^{2} /\left(\mu_{2} U\right)$ defines the ratio of gravitational to viscous forces and can be considered a measure of the density contrast between the two fluids. Here $g$ denotes the gravitational acceleration in the $-x$ direction. Positive $F$-values (heavier fluid on top) correspond to gravitationally unstable displacements, whereas for $F<0$ (lighter fluid on top) gravity is stabilizing.

Our emphasis will be on exploring the influence of the parameter $F$ over a range of values from -100 to 100 . In addition, we will present simulation results for several different values of $P e$ between 500 and 5000. The competition between the inner splitting and tip-splitting instabilities will be investigated by varying the spanwise extent of the domain, along with the form of the initial perturbation. Except for validation purposes, the viscosity ratio will be kept constant at $M=3$, and the Reynolds number will be fixed at $R e^{*}=1$. We point the reader to Oliveira \& Meiburg (2011) for a detailed discussion of the effects of $M$ and $P e$.

In order to relate these dimensionless parameters to typical laboratory experimental set-ups, let us consider the following dimensional quantities. Water as the less-viscous 
fluid and an aqueous solution of $70 \%$ glycerine in weight as the more-viscous fluid at $20^{\circ} \mathrm{C}$ yield a dimensionless viscosity ratio close to $M=3$. The diffusivity of these two fluids can be obtained from D'Errico et al. (2004) and Rashidnia \& Balasubramaniam (2004), and $D$ falls in the range from $0.07 \times 10^{-9}$ to $0.2 \times 10^{-9} \mathrm{~m}^{2} \mathrm{~s}^{-1}$. For Hele-Shaw cells with gap thickness $b$ ranging from 0.5 to $1.5 \mathrm{~mm}$ and an injection flow rate with characteristic velocity $U$ between 0.25 and $2.5 \mathrm{~mm} \mathrm{~s}^{-1}$, Péclet numbers in the interval [650, 56000] are obtained. Similarly, the ranges of the Reynolds numbers and positive gravity numbers are found to be $R e \in[0.1,3.7]$ and $F \in[8,710]$, respectively. This shows that our choice of dimensionless quantities corresponds to typical laboratory experiments.

\section{Numerical approach}

Following Oliveira \& Meiburg (2011), we employ a fractional-step method as proposed by Rai \& Moin (1991), based on the time-split scheme of Kim \& Moin (1985), to solve (2.1)-(2.3) on a staggered grid. This scheme uses finite differences in a three-step hybrid Runge-Kutta/Crank-Nicolson discretization. An explicit iterative Runge-Kutta method is used for approximating the convective terms, whereas an implicit Crank-Nicolson method is used for the viscous terms. Thus, the method is second-order accurate in time for the viscous terms and third-order accurate in time for the convective terms, rendering the method's overall accuracy second-order in time. Note that the two-dimensional simulations of $\S \S 4.1$ and 5 were performed using the three-dimensional code, by keeping three grid points in the spanwise direction; this is the minimum number of points necessary to resolve the WENO scheme employed in the discretization of the convective terms. To avoid repetition, we refer the reader to Oliveira \& Meiburg (2011) for details of the spatial and temporal discretization and of the numerical implementation.

\subsection{Initial conditions}

The initial condition prescribes a Poiseuille-type inflow for the $u$ velocity component:

$$
u(x=0, y, z)=1.5 \cdot\left(1-4 y^{2}\right) .
$$

The initial concentration distribution is determined by an error-function profile centred at $x=x_{\text {init }}$, defined as

$$
c(x, y, z)=0.5+0.5 \cdot \operatorname{erf}\left(\frac{x-x_{\text {init }}}{\delta}\right) .
$$

As previous investigations have found the quasisteady shape of the two-dimensional displacement front to be independent of its initial thickness (Goyal \& Meiburg 2006), the parameter $\delta$ is set to 0.1 . Additionally, the initial interface position $x_{\text {init }}$ is set to sufficiently large values to enable us to fully resolve the evolving reversed flow, as will be explained in more detail below.

\subsection{Boundary conditions}

We take advantage of the inherent double-symmetry of the flow to drastically reduce the size of the computational domain and the memory and processor requirements. However, we note that this symmetry assumption will not allow us to capture potential 
symmetry-breaking instabilities. Along the solid wall at $y=b / 2$, we enforce no-slip conditions and a vanishing wall-normal gradient of $c$, implying the absence of a concentration flux across the wall. The spanwise boundary of the computational domain is implemented as a slip symmetry plane using one ghost point, requiring the velocity across the plane, $u_{\perp}$, and all other gradients perpendicular to the plane to vanish (see figure 1). The Poiseuille profile as defined in (3.1) at the inflow boundary $x=0$ forces a constant supply of less-viscous fluid $(c=0)$ into the cell. A purely convective outflow condition

$$
\frac{\partial \zeta}{\partial t}+U_{\max } \frac{\partial \zeta}{\partial x}=0
$$

is imposed on the ghost nodes at the outflow boundary for $\zeta=(u, v, w, c)$, with $U_{\max }$ being the maximum $u$ velocity at the outflow surface.

\subsection{Adaptive domain size}

In addition to parallelizing the direct numerical simulations (DNS) code by means of domain decomposition along the main flow direction, a further significant speedup can be obtained by employing an adaptive domain size as the displacement front evolves in the streamwise direction. This requires the careful tracking and localization of areas of flow that deviate from the undisturbed Poiseuille flow field downstream of the front. The simulation is halted as soon as sufficiently strong gradients are detected upstream of the outflow. Subsequently, the domain size is increased by inserting additional subdomains upstream of the outflow boundary. By allowing the domain to grow in time in this way, the CPU time is drastically decreased.

\section{Validation}

\subsection{Properties of the displacement front}

Here we extend the validation of the DNS code performed by Oliveira \& Meiburg (2011) for the neutrally buoyant case, by reproducing the linear stability results of Goyal et al. (2007) for purely two-dimensional Stokes flows. The two-dimensional quasisteady base state that develops after an appropriate period of time exhibits a sharp displacement front followed by diffusively spreading lateral concentration layers (Chen \& Meiburg 1996); see figure 2.

This displacement front is characterized by its thickness $d$ and tip velocity $u_{\text {tip }}$. Following Oliveira \& Meiburg (2011), we define $d$ to be the vertical distance between the $c=0.1$ and $c=0.9$ concentration contours along the centre-line of the Hele-Shaw cell $y=z=0$, and we denote by $u_{t i p}$ the propagation velocity of the $c=0.5$ contour along this line; $d_{0}$ and $u_{t i p, 0}$ indicate quasisteady values that are reached after an initial transient period. Figure 3 plots $d_{0}$ and $u_{t i p, 0}$ as functions of the gravity parameter $F$ for various mobility ratios $M$. The corresponding base-flow results reported by Goyal et al. (2007) for Stokes flows are reproduced for comparison. The agreement between the numerical results and the reference values is reasonable, with relative differences for $d_{0}$ between 0.3 and $8.1 \%$, and those for $u_{t i p, 0}$ in the range $0.06-1.7 \%$.

\subsection{Instability growth rates}

Goyal et al. (2007) performed a linear stability analysis of the above two-dimensional quasisteady base flows with respect to wave-like perturbations in the spanwise 


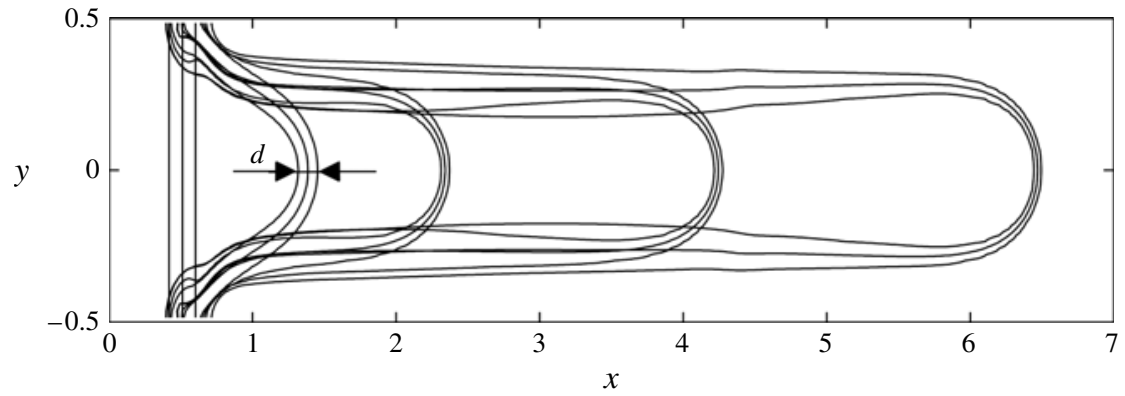

FIgURE 2. Temporal evolution of the two-dimensional upward base-flow displacement front for $M=3, F=20, P e=2000$ and $R e^{*}=1$. The contours $c=0.1,0.5$ and 0.9 of the concentration field are plotted at times $t=0,0.5,1,2$ and 3 . The front thickness $d$ is computed as the vertical distance between the $c=0.1$ and $c=0.9$ layers along the centre-line of the cell.

(a)

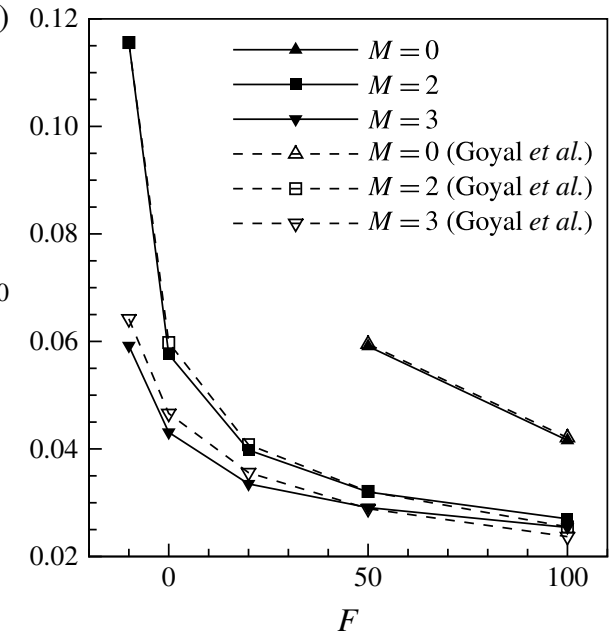

(b)

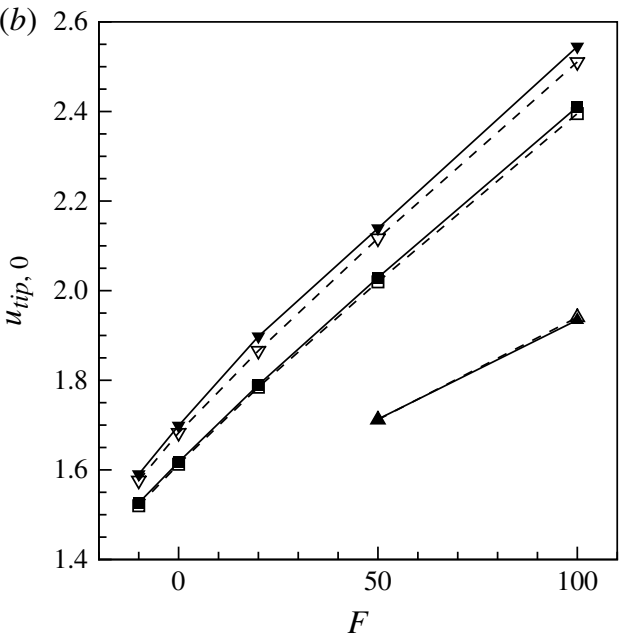

FIGURE 3. Plots as functions of the gravity parameter $F$ of $(a)$ the quasisteady front thickness, $d_{0}$, and $(b)$ the quasisteady tip velocity, $u_{t i p, 0}$, of the two-dimensional displacement front for different viscosity ratios $M$, with $P e=2000$ and $R e^{*}=1$. We find good agreement between the current Navier-Stokes simulation results and the corresponding Stokes values computed by Goyal et al. (2007).

direction. Their predicted growth rates for the most unstable mode can be compared to growth rates extracted from the present three-dimensional fully nonlinear simulations. For this purpose we performed simulations for various combinations of $M \in\{0,2,3\}$ and $F \in\{0,20,50,100\}$, keeping $P e=1000$ and $R e^{*}=\rho_{1} U b / \mu_{1}=1$ constant. Goyal et al. (2007) had found that for displacements governed by both viscosity and density contrasts, both the growth rate and the wavenumber of the most unstable mode depend only weakly on the Péclet number. This allows us to compare growth rate data from our nonlinear simulations for $P e=1000$ with the linear stability data of Goyal et al. (2007) for $P e=2000$. 


$\begin{array}{ccccr}M & F & \sigma_{D N S} & \sigma_{L S A} & \epsilon(\%) \\ 0 & 50 & 1.218 & 1.351 & -9.845 \\ 0 & 100 & 2.620 & 3.069 & -14.630 \\ 2 & 0 & 0.343 & 0.347 & -1.153 \\ 2 & 20 & 1.627 & 1.707 & -4.687 \\ 2 & 50 & 3.600 & 3.827 & -5.932 \\ 2 & 100 & 6.716 & 7.430 & -9.610 \\ 3 & 0 & 0.799 & 0.760 & 5.132 \\ 3 & 10 & 1.664 & 1.606 & 3.579 \\ 3 & 20 & 2.532 & 2.458 & 3.011 \\ 3 & 30 & 3.379 & 3.332 & 1.400 \\ 3 & 50 & 4.978 & 5.042 & -1.269 \\ 3 & 100 & 8.615 & 9.385 & -8.205\end{array}$

TABLE 1. Comparison of linear growth rates $\sigma_{D N S}$ computed from the present NavierStokes simulations with the corresponding linear stability analysis growth rates $\sigma_{L S A}$ obtained by Goyal et al. (2007) for Stokes flows. The parameter values are $P e=1000$, $M=0,2$ or 3 and $F=0,10,20,30,50$ or $100 ; \epsilon$ represents the discrepancy between the growth rates, reported as a percentage.

After the base flow is fully developed and a quasisteady state is reached, a wavelike perturbation of the most unstable wavelength $\lambda_{m}$ (as predicted by linear stability analysis) of small amplitude is added to the concentration field. We note that the size of the spanwise domain matches the wavelength of the imposed perturbation. This superposed disturbance shifts the concentration field according to

$$
c(x, y, z) \rightarrow c\left(x+\hat{A} \cos \left(\frac{2 \pi}{\lambda_{m}} z\right), y, z\right) .
$$

The amplitude $\hat{A}$ is chosen sufficiently small $\left(\hat{A}=1 \times 10^{-3}\right)$ (Oliveira \& Meiburg 2011) to ensure that the perturbation will grow linearly over an extended time interval. The growth rate of the perturbation is then computed by extracting the maximum absolute value of the spanwise velocity component $|w|_{\max }$ in the entire flow field and then applying a least-squares regression fit to a selected temporal range of the data using built-in MATLAB routines and optimization functions.

A comparison of the growth rates $\sigma_{D N S}$ obtained from the DNS with the linear stability analysis data $\sigma_{L S A}$ of Goyal et al. (2007) is presented in table 1. Generally, the agreement improves with increasing $M$ and, for small $M$, with decreasing $F$-values. While the precise reason for this influence of $M$ and $F$ on the level of agreement is not known, the discrepancies are mostly below $10 \%$, which can be considered satisfactory.

\section{Two-dimensional base flow}

The displacement of a viscous fluid by another fluid of lower viscosity has been the subject of numerous investigations. Taylor (1961) studied the immiscible displacement of a viscous fluid by injecting air into a horizontal capillary tube. Following Fairbrother \& Stubbs (1935), Taylor related the film thickness of displaced fluid left behind on the wall to a quantity $m$, defined by $m=1-U / u_{\text {tip }}$, and measured its dependence on the capillary number $\mathrm{Ca}=U \mu_{2} / \sigma$. Here, $\sigma$ denotes the surface tension, while all other variables are defined as above. Furthermore, Taylor proposed 
different streamline patterns outside the finger, in a reference frame moving with the finger tip, as functions of $m$ and thus of the propagation velocity of the finger.

Petitjeans \& Maxworthy (1996) performed experiments extending Taylor's investigations to miscible displacements in capillary tubes. The usage of two miscible fluids, glycerine and glycerine-water mixtures, allowed them to vary the viscosity and density differences, and in turn the dominant parameters $P e, F$ and a viscous Atwood number $A t=\left(\mu_{2}-\mu_{1}\right) /\left(\mu_{2}+\mu_{1}\right)$, over an extended range. Petitjeans \& Maxworthy (1996) extended the streamline patterns originally proposed by Taylor (1961) to the flow inside the finger. Both works suggested that for $m>0.5$ the flow resembles a stagnation-point flow with a single stagnation point located at the tip of the finger. For $m<0.5$, Petitjeans \& Maxworthy (1996) showed that the stagnation point at the tip is complemented by an additional stagnation point inside the finger, yielding the existence of a closed off-axis stagnation ring on the finger surface, consistent with Taylor's hypothesized pattern. Due to the absence of surface tension and the effect of diffusion, a 'spike' can grow from the tip of the finger.

In conjunction with these experimental investigations, numerical simulations of displacement flows in capillary tubes and plane channels were performed by Chen $\&$ Meiburg (1996). These simulations confirm some of the above patterns, and the authors furthermore discuss the effects of unsteadiness. Lajeunesse et al. (1999) conducted experimental investigations of miscible displacements in vertical cylindrical tubes and Hele-Shaw cells. They, too, were able to observe the growth of the 'needle' or 'spike'-shaped geometry for favourable density stratifications. In addition, Goyal et al. (2007) observed the evolution of this two-dimensional phenomenon for Hele-Shaw displacements by means of computational simulations but did not investigate the corresponding streamline patterns in depth.

As a first step, we investigate the two-dimensional base flow in the $(x, y)$-plane before the onset of the three-dimensional instability. For corresponding miscible displacements in axisymmetric capillary tubes, Chen \& Meiburg (1996) pointed out that a quasisteady state will develop only in certain parameter regimes, and it will persist for only a limited period of time. This limitation is primarily due to molecular diffusion, which eventually renders even the local flow near the displacement front time-dependent. In the following, we provide an overview of the different types of base flows and respective streamline topologies that can evolve by discussing the representative, gravitationally stable parameter values $F=0,-20$ and -60 , for $M=3$, $P e=1000$ and $R e^{*}=1$.

For the neutrally buoyant case $F=0$, the two-dimensional simulations in figure 4 indicate a steadily advancing front. Unless otherwise noted, in this and all following concentration plots the levels of the concentration contours are $c=0.1,0.2, \ldots, 0.9$. Within the finger and behind the front, concentration contours undergo a continuous 'pinch-off' on the symmetry line $y=0$, starting with the smallest $c$-values and affecting successively larger $c$-values, which results in the spreading of the displacement front.

The streamline pattern in the reference frame moving with the instantaneous propagation velocity $u_{t i p}$ of the finger tip, i.e. with the velocity of the $c=0.5$ contour along the centre-line of the cell, is illustrated in figure $4(b)$ for time $t=10$. The streamlines indicate a stagnation-point flow at the tip, which compresses the miscible interface in the streamwise direction. The pattern outside the interface and the zero streamline compare well with the streamlines hypothesized by Taylor (1961) for $m>0.5$ in axisymmetric flows. Two-dimensional Poiseuille flow has a maximum velocity of 1.5 , as opposed to the value of 2 for axisymmetric flow, which leads to a critical value of $m_{\text {crit }}=1 / 3$ instead of $m_{\text {crit }}=0.5$, implying a critical tip velocity of 
(a)
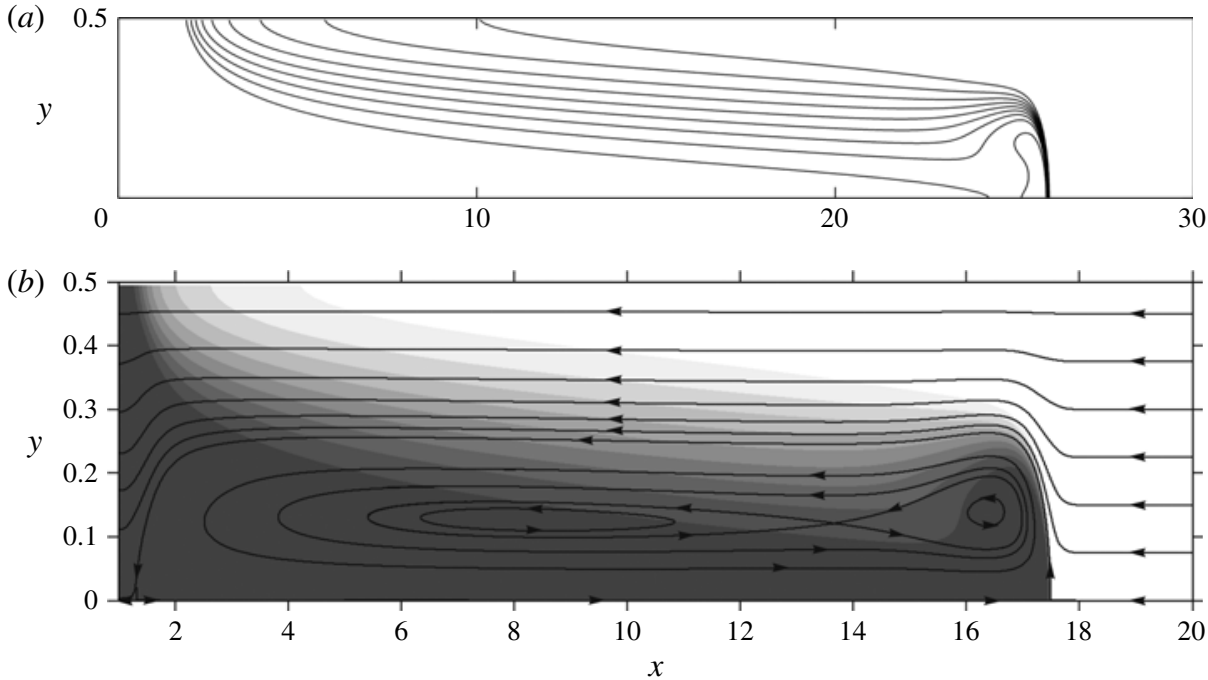

Figure 4. (a) Concentration field at time $t=15$, in which the lines represent $c=$ $0.1,0.2, \ldots, 0.9$, for $M=3, F=0, P e=1000$ and $R e^{*}=1$; note the pinch-off of the $c=0.1$ concentration contour in the interior of the finger behind the front. (b) Concentration contours in greyscale shading together with the streamline pattern in the moving reference frame at time $t=10$; as the propagation velocity of the tip is larger than the maximum Poiseuille flow velocity of 1.5 , a stagnation point exists on the centre-line at the tip.

$u_{\text {tip,crit }}=1.5$. Thus, a propagation velocity of the tip that is larger than $u_{\text {tip,crit }}$ leads to the flow topology shown in figure $4(b)$.

Figure 5(a) illustrates the temporal evolution of the pinch-off as a function of the gravitational parameter $F$. Because the moment at which the pinch-off occurs is calculated during the postprocessing stage, the error associated with these measurements is of the order of the time interval between data outputs, which is $\Delta t=0.1$. We note a very weak delay of the pinch-off for a given concentration level with increasing $F$-values, even though the front propagates faster and the corresponding vorticity field is stronger. On the other hand, figure $5(b)$ demonstrates a strong dependence of the pinch-off on the Péclet number. This indicates that the pinch-off phenomenon is dominated by diffusive effects rather than by the vorticity field.

Figure $6(a)$ shows the concentration field for $c=0.1,0.2, \ldots, 0.9$ with parameters $M=3, F=-20$ and $P e=1000$ at time $t=10$. The negative $F$-value implies gravitationally stable behaviour. Figure $6(b)$ illustrates the corresponding topological nature of the streamline pattern for these parameters. The pattern is clearly different from the one found for $m>1 / 3$ (cf. figure $4 b$ ) and agrees with the scenario suggested for $m<0.5$ by Taylor (1961) and Petitjeans \& Maxworthy (1996) for miscible displacements. A remarkable feature is the absence of a stagnation point on the centre-line, so that less viscous fluid is able to propagate along the centre-line and form a protrusion. Since the streamline pattern is shown in the reference frame moving with the centre-line velocity of the $c=0.5$ contour, the absence of a stagnation point indicates that the fluid velocity at the location of the $c=0.5$ contour is larger than the velocity with which the contour itself propagates. This velocity difference is due to the effects of diffusion. We remark that for neutrally buoyant flows with $m$ below 
(a)

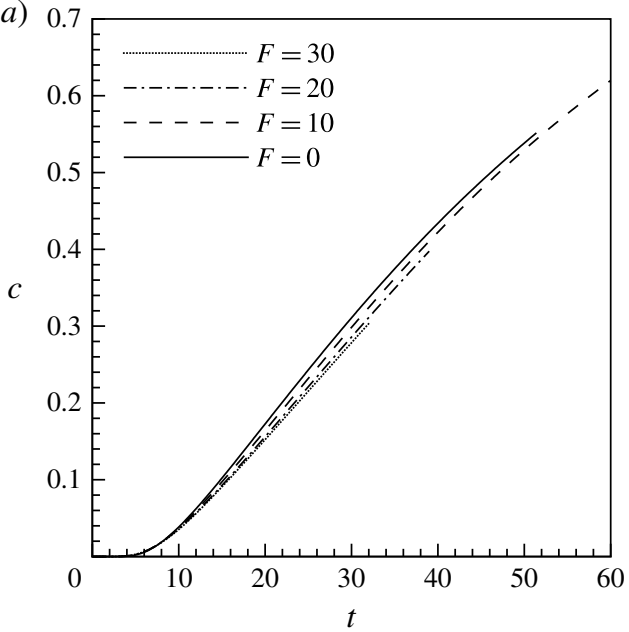

(b)

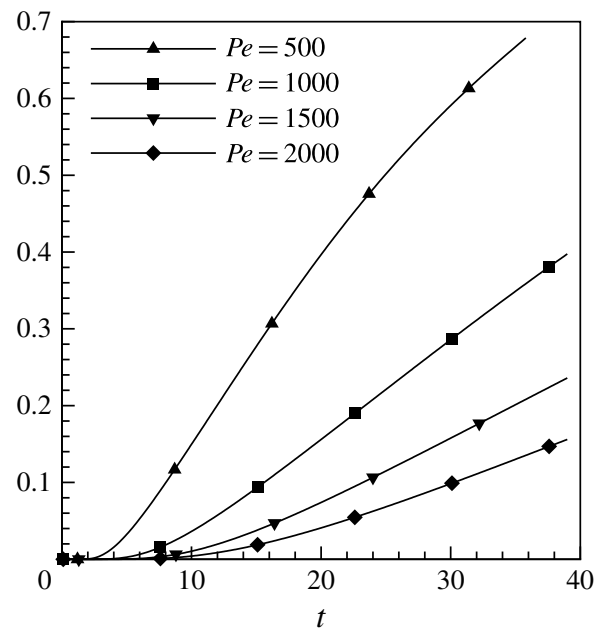

FIGURE 5. Evolution of the pinch-off for continuous concentration levels as a function of time $t$ : (a) with $M=3, R e^{*}=1, P e=1000$ and gravity numbers $F=0,10,20$ and 30; (b) with $M=3, R e^{*}=1, F=20$ and Péclet numbers $P e=500,1000,1500$ and 2000. Diffusive effects are seen to dominate the pinch-off mechanism.

the critical value, Oliveira \& Meiburg (2011) showed that the fluid velocity at the location of the $c=0.5$ contour is smaller than the propagation velocity of the contour. In this way, the displacement front is able to develop a 'needle'- or 'spike'-shaped geometry, with a second mechanism to be discussed below. As pointed out by Kuang et al. (2004), the continuous leakage of less-viscous fluid from the displacement front implies that these fronts are never quasisteady.

A second notable feature of the streamline pattern is the existence of an off-axis saddle point whose precise position is a function of the difference between $u_{\text {tip }}$ and the maximum Poiseuille flow velocity of 1.5. The steep concentration front located on the side of the tip owes its existence to the presence of the recirculation region behind the front, which transports fluid from the centre-line towards larger $y$-values, thus sharpening the concentration gradient.

A further decrease of the gravity number $F$ leads to additional changes in the topology of the flow field. Figure 7 shows the temporal evolution of the concentration field for $M=3, F=-60, P e=1000$ and $R e^{*}=1$ at times $t=2,4,6,7$ and 10 . The growth of the 'spike' and its spatial extent are more pronounced than for moderate negative gravity numbers. The deformation of the concentration field closely behind the tip resembles that reported by Petitjeans \& Maxworthy (1996) and confirmed by Chen \& Meiburg (1996) for axisymmetric flows, which they called a 'plume'.

Compared to the case of $F=-20$, the streamline pattern in the reference frame moving with the $c=0.5$ contour has undergone additional bifurcations, which increase the complexity of the flow topology; see figure 8. First, a stagnation point can be found in the tip region on the centre-line of the cell, which is in contrast to the observations of Chen \& Meiburg (1996) for axisymmetric displacements with comparably high negative gravity numbers. Note that the flow in the vicinity of this stagnation point is reversed as compared to the $F=0$ case shown in figure $4(b)$. This stagnation-point flow spreads the front in the upstream and downstream directions. In addition, the small recirculation zone immediately behind the tip of the displacement 

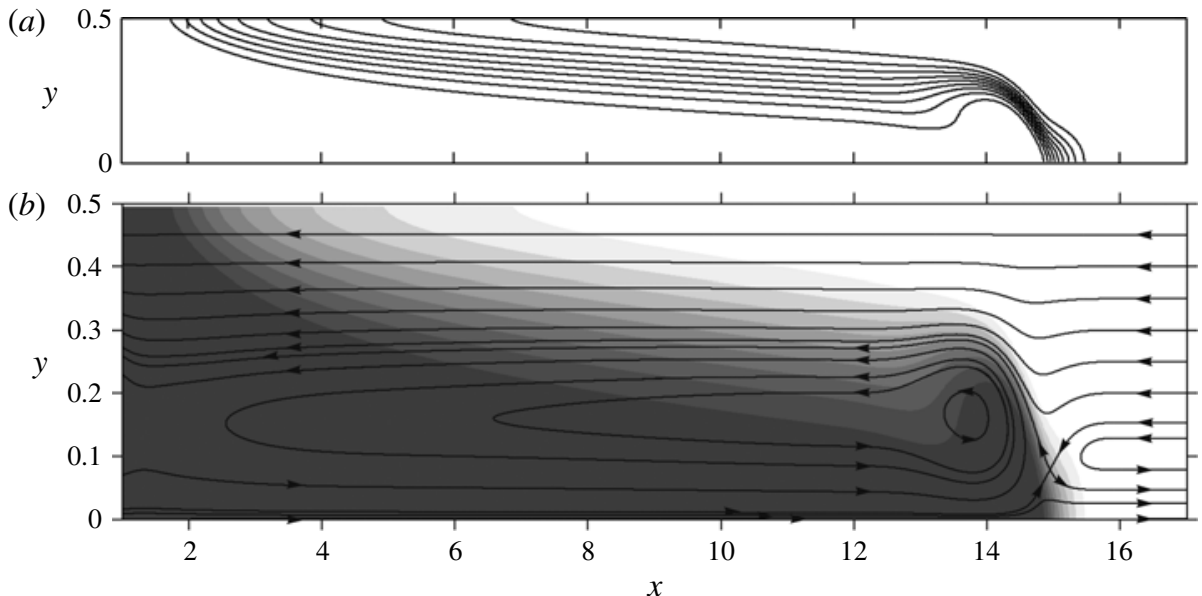

Figure 6. (a) Concentration contours $c=0.1,0.2, \ldots, 0.9$ for $M=3, F=-20, P e=$ 1000 and $R e^{*}=1$ at time $t=10$ : a protrusion is beginning to form at the finger tip. (b) The same concentration contours in greyscale shading together with the corresponding streamline pattern in the moving reference frame.

(a)

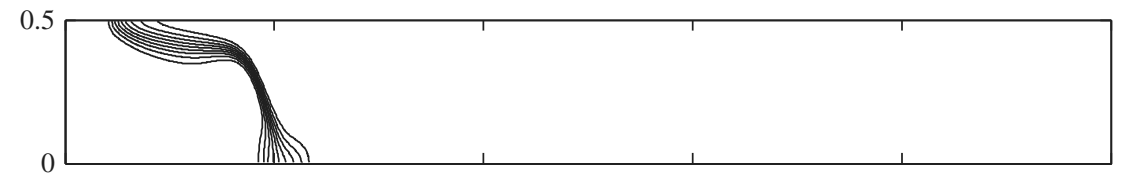

(b)

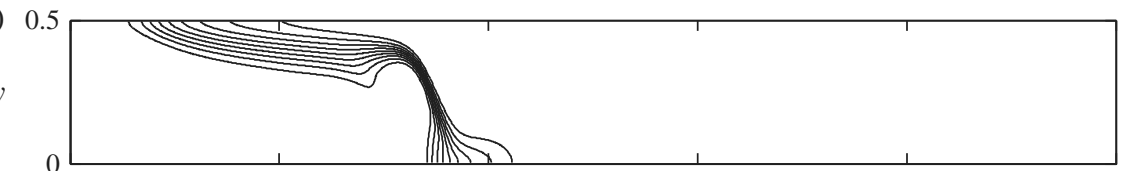

(c)
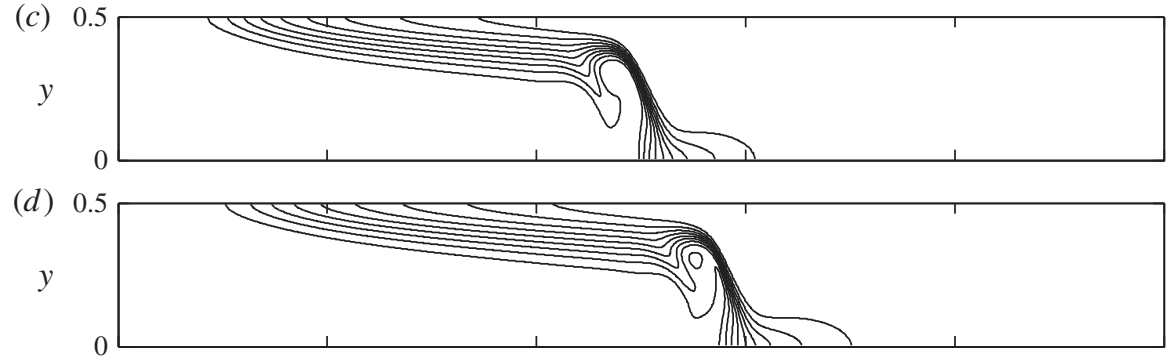

(e)

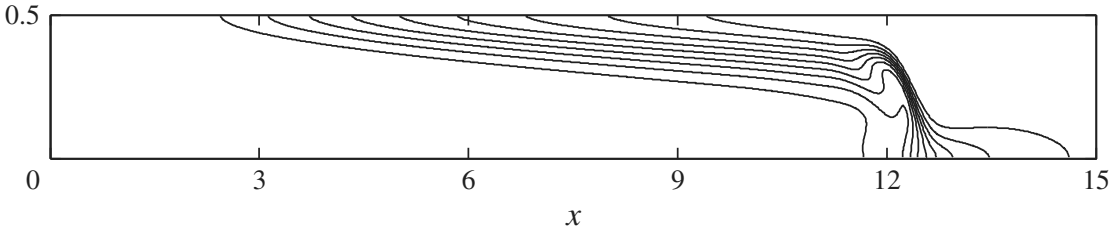

FIgURE 7. Evolution of the concentration field for $M=3, F=-60, P e=1000$ and $R e^{*}=$ 1 ; the times shown are (a) $t=2,(b) t=4,(c) t=6,(d) t=7$ and $(e) t=10$. The contour lines represent $c=0.1,0.2, \ldots, 0.9$. 


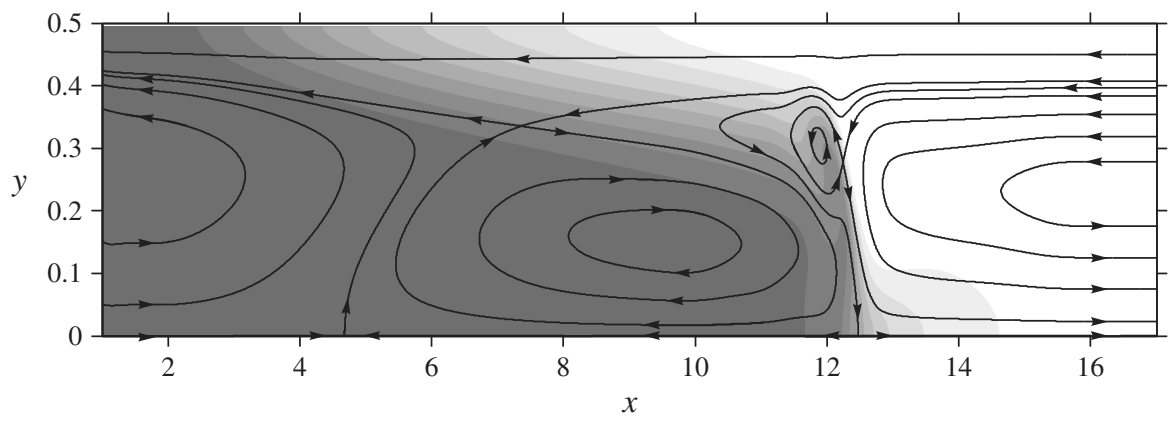

FIGURE 8. Concentration contours $c=0.1,0.2, \ldots, 0.9$ in greyscale shading, together with the corresponding streamline pattern in the moving reference frame, for $M=3, F=-60$, $P e=1000$ and $R e^{*}=1$ at time $t=10$.

front has shifted to larger $y$. Note that the 'spike' is no longer formed by leakage of less-viscous fluid through the tip along the centre-line. Rather, it now owes its evolution to the presence of a channel of complex geometry supplying the 'spike' with less-viscous fluid.

As pointed out by Petitjeans \& Maxworthy (1996), Alba, Taghavi \& Frigaard (2012) and other authors, this 'spike'-shaped structure has been observed only in miscible displacements, whereas in immiscible flows surface tension would probably keep it from evolving. Figure 9 compares this structure for $\left(M, F, R e^{*}\right)=(3,-60,1)$ and increasing values of the Péclet number $(P e=500,1000,2000$ and 5000) at time $t=10$. An increase in $P e$ has a very weak influence on the length of the structure, whereas the width becomes progressively thinner. It appears possible that in the limit where no diffusion is present, the 'spike'-shaped structure will not evolve.

For larger times, the flow field becomes increasingly complex, as can be seen in figure 10(a). Immediately behind the strongly diffused tip a region has emerged that is characterized by oscillatory concentration contours. The corresponding streamline pattern in the moving reference frame is illustrated in figure 10(b). A series of co-rotating vortices dominates the flow behind the tip. These vortices transport less-viscous fluid in the downstream direction into the 'spike', while also causing alternating lateral transport, thereby generating the oscillations in the concentration contours and associated mixing.

Figure 11(a) displays the displacement front position, measured along the centre-line $y=0$, as a function of time for different values of the viscosity ratio and gravity number. These plots indicate that quasisteady values of the front velocity $u_{t i p}$ can be estimated. Representative values of $u_{t i p}$ are displayed in figure $11(b)$ as a function of the gravity number $F$, for different values of $M$ and $P e$. As discussed above, the displacement is a transient phenomenon, so truly steady propagation velocities are not achieved. The values shown in this plot represent the average propagation velocities of the tip up to the time at which the pinch-off of the $c=0.1$ concentration contour occurs. During this early stage, $t=O(10)$, unsteady effects are weak, and the reported velocity values of figure $11(b)$ are steady over at least a couple of units in time with a precision of the order of $1 \%$. These average velocities allow us to discuss the effects of $F$ and $M$ on the displacements. With decreasing gravity number, the tip velocity deviates from the nearly linear regime found for gravitationally unstable flow; cf. figure 3. This observation is in contrast to the axisymmetric results of 


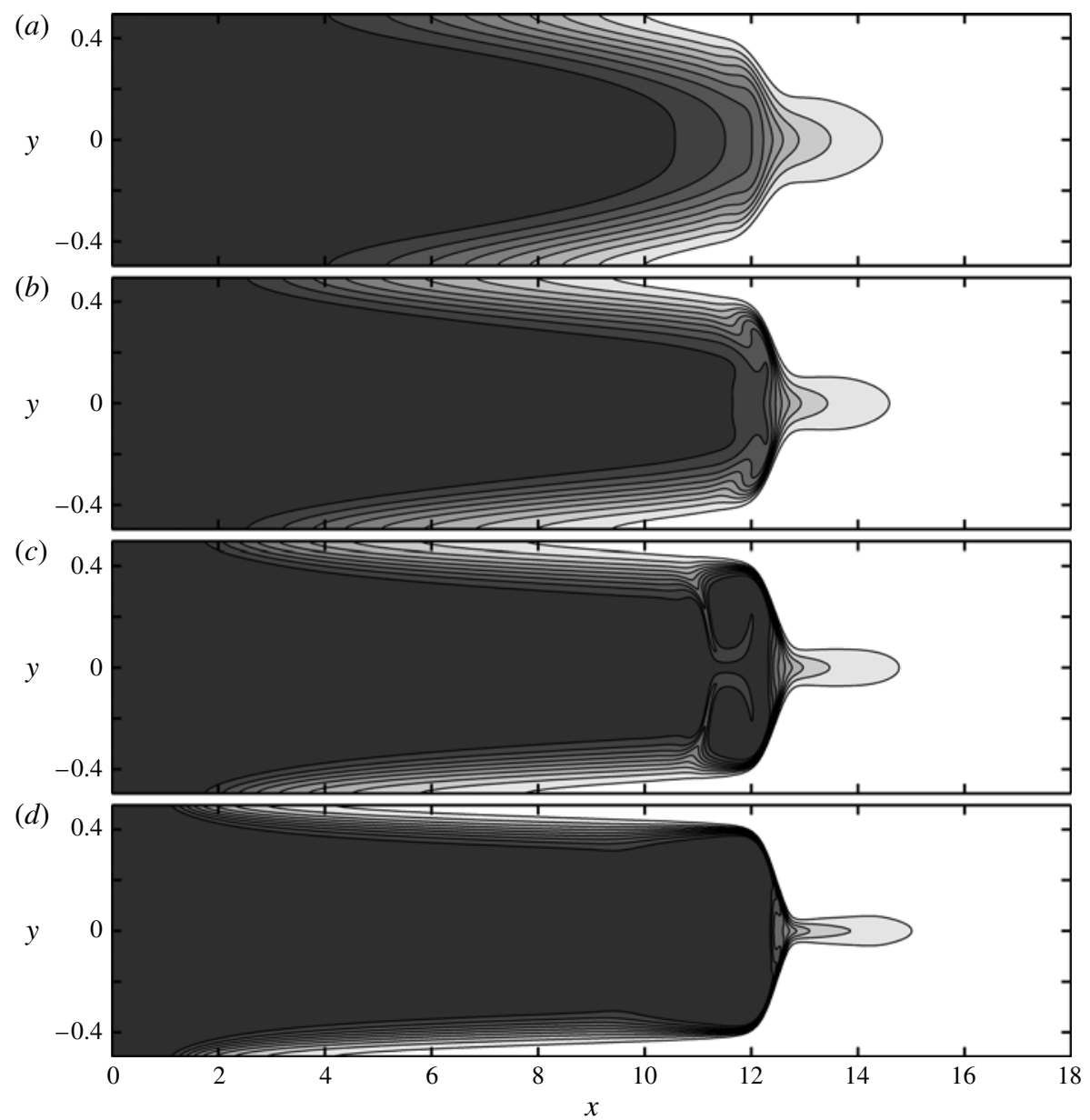

FIgURE 9. Concentration field in grey, with contours in increments of $\Delta c=0.1$, for $\left(M, F, R e^{*}\right)=(3,-60,1)$ at time $t=10:$ (a) $P e=500$, (b) $P e=1000$, (c) $P e=2000$ and $(d) P e=5000$. Larger $P e$-values lead to thinner 'spikes'.

Chen \& Meiburg (1996), who found the linear relationship between $u_{\text {tip }}$ and $F$ to extend into the gravitationally stable regime. Furthermore, the values for $F=0$ are in excellent agreement with the numerical results on miscible displacements between parallel plates obtained by Rakotomalala, Salin \& Watzky (1997).

\section{Three-dimensional evolution}

Much of the previous section on two-dimensional base flows focused on the gravitationally stable regime, $F<0$, where the formation of spikes results in interesting dynamics. However, the linear stability results of Goyal et al. (2007) show that this regime is largely stable with respect to spanwise perturbations. For this reason, the three-dimensional simulations described below will focus on the gravitationally unstable regime $F>0$, where pronounced fingering occurs.

We begin by discussing the representative case with $M=3, F=20, P e=1000$ and $R e^{*}=1$. Figure 12 shows a perspective view of the concentration isosurface 
(a)

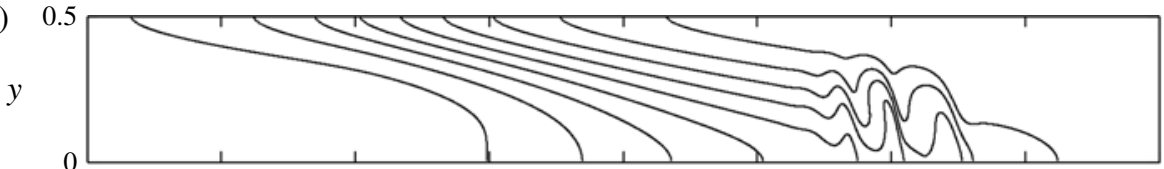

(b)

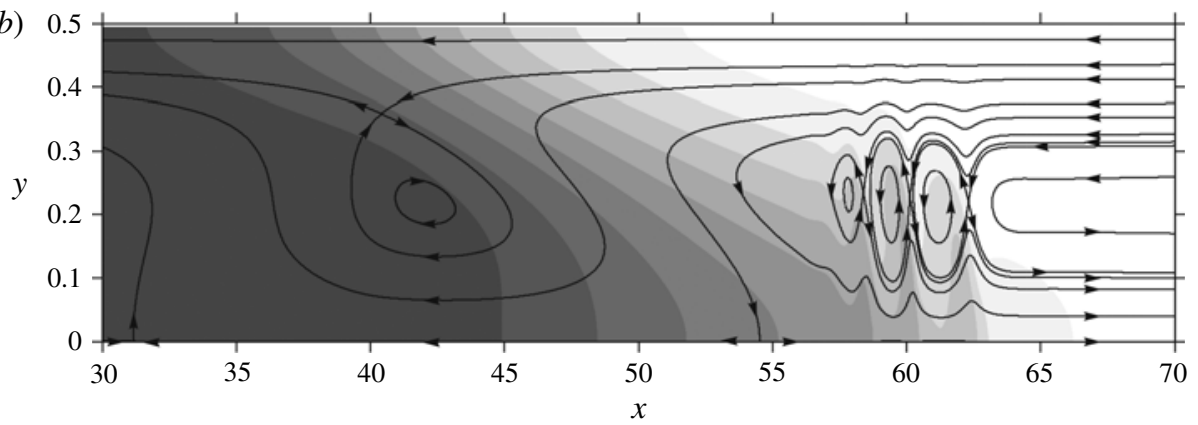

Figure 10. (a) Concentration contours $c=0.1,0.2, \ldots, 0.9$ for $M=3, F=-60$, $P e=1000$ and $R e^{*}=1$ at time $t=50$; oscillatory contours are observed behind the tip. (b) The same concentration contours in greyscale shading together with the corresponding streamline pattern in the moving reference frame; co-rotating vortices are observed near the oscillatory contours.

$c=0.35$ at times $t=20$ and 38. For clarity, two spanwise wavelengths are shown. The flow field was initialized in a two-dimensional fashion as described in $\S 3$, i.e. with an error-function profile for the concentration distribution centred around $x_{\text {init }}=40$ and a Poiseuille flow velocity field. This velocity field instantly deforms the concentration field and leads to the formation of a two-dimensional quasisteady displacement front, which is observed beginning at approximately time $t=2$. A wavy spanwise perturbation of the linearly most unstable mode with an amplitude of $\hat{A}=0.03$ is then added at $t=2$ to the concentration field as given by relation (4.1). While the amplitude of the imposed perturbation determines the time at which the observed nonlinear flow features emerge, the nature of these features is largely independent of the imposed perturbation amplitude. Subsequently, the flow evolves fully three-dimensionally. The spanwise domain size $L_{z}$ is set equal to the wavelength of the imposed perturbation.

Initially a long, smooth, upward-propagating finger of light fluid forms, similar to the neutrally buoyant case analysed by Oliveira \& Meiburg (2011). Driven by the unstable density stratification, however, an additional downward-propagating dense front emerges as well, which eventually takes on a characteristic anchor-like shape; see figure 12(b). At this late time, the upward-propagating finger composed of light fluid is seen to have undergone an inner splitting instability, in a manner very similar to the neutrally buoyant case. This suggests the presence of a quadrupole vortex structure in the interior of the finger.

Such a vortex structure is confirmed by the $x=$ constant cross-cuts shown in figure 13 for time $t=38$. Panel $(a)$ shows the region immediately behind the tip of the upward-propagating finger of light fluid. An inner quadrupole vorticity structure is seen to convect resident, viscous fluid from the solid walls towards the centre of the cell near $z=0$. Simultaneously, injected less-viscous fluid is transported laterally away from the centre along $y=0$. In this fashion, an inner splitting event results that is qualitatively similar to what occurs in the neutrally buoyant case. An additional 

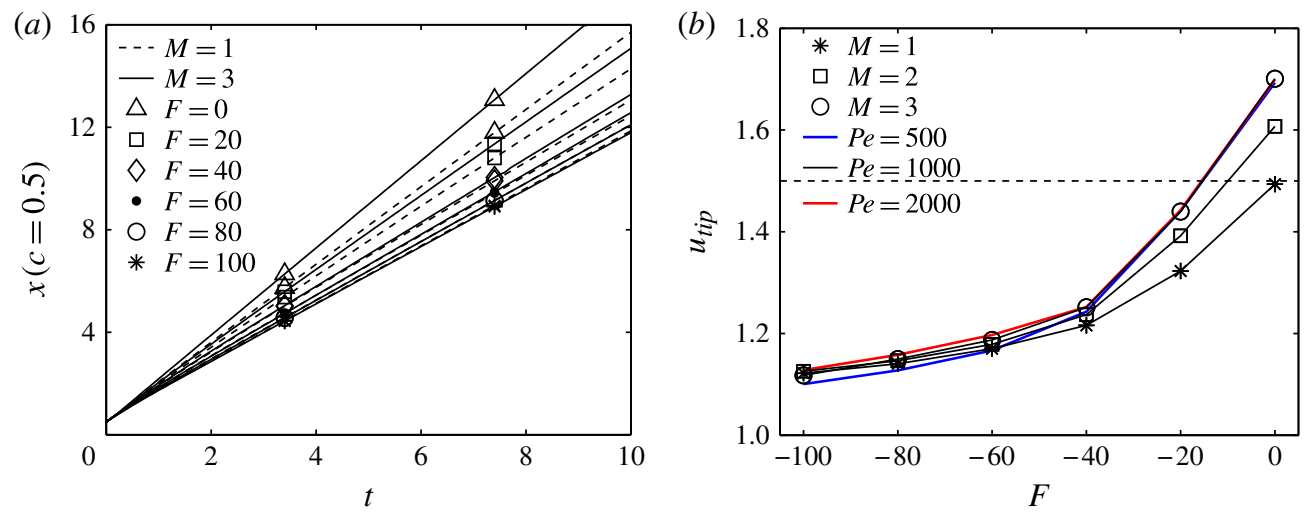

FIgURE 11. (Colour online) (a) Front position $x(c=0.5)$ of the displacement along the centre-line as a function of time for $P e=1000$ and different values of $M$ and $F$. (b) Quasisteady front velocity $u_{t i p}$ as a function of the gravity number $F$ for different values of $M$ and $P e$; the non-black lines are plotted for $M=3$, and the horizontal dashed line represents the maximum Poiseuille velocity of 1.5 .

weaker, counter-rotating quadrupole vorticity structure can be recognized closer to the four corners of the domain. For smaller $x$-values, these counter-rotating vortices gain in strength - compare $(b)$ and $(c)$ - and eventually dominate over the central quadrupole structure. Panel $(d)$ shows that the streamwise vortex structure within the downward-propagating anchor region is qualitatively similar, in that it also exhibits inner and outer quadrupole vortices. However, these quadrupoles are now centred near the spanwise symmetry boundaries $z= \pm L_{z} / 2$, whereas the quadrupole in the upward-propagating finger is centred near $z=0$. The anchor quadrupole is responsible for injecting less-viscous fluid between the stem of the anchor and its flukes. This will be discussed in more detail below.

Figure 14 shows the temporal evolution of the leading and trailing front locations and velocities. As expected, stronger unstable density stratifications result in more rapidly upward-propagating fingers, as can be seen in $(a)$. Figure 14(b) illustrates the evolution of the tip velocity $u_{t i p}=\mathrm{d} x_{t i p} / \mathrm{d} t$. Upon introduction of the spanwise perturbation at $t=2$, the finger tip rapidly accelerates, attains a maximum velocity, and then gradually slows down again.

The location $x_{s}$ of the dense, trailing front can be defined in analogy to the upward-propagating finger tip location $x_{\text {tip }}$. Figure $14(c)$ illustrates its temporal evolution for different values of $F$, and figure $14(d)$ shows the corresponding propagation velocity $u_{s}=\mathrm{d} x_{s} / \mathrm{d} t$. In the neutrally buoyant case, the trailing front is seen to propagate upward with nearly constant velocity. As we increase the unstable density stratification, the dense front gradually slows down, eventually reversing direction and propagating downward. Note that the results shown in figure $14(c, d)$ suggest that there may exist an $F$-value such that the trailing front will remain stationary for some time. The downward-propagating fronts exhibit strongly time-dependent propagation velocities, which reflects the various phases of anchor formation, as will be discussed below. Goyal et al. (2007) found that the growth rate of the most unstable wavenumber depends only weakly on $\mathrm{Pe}$. We expect that $u_{s}$ also depends weakly on $P e$.

In order to gain insight into how the inner quadrupole's strength depends on the magnitude of $F$, we first find the maximum value $\Omega_{x}$ of the streamwise vorticity 


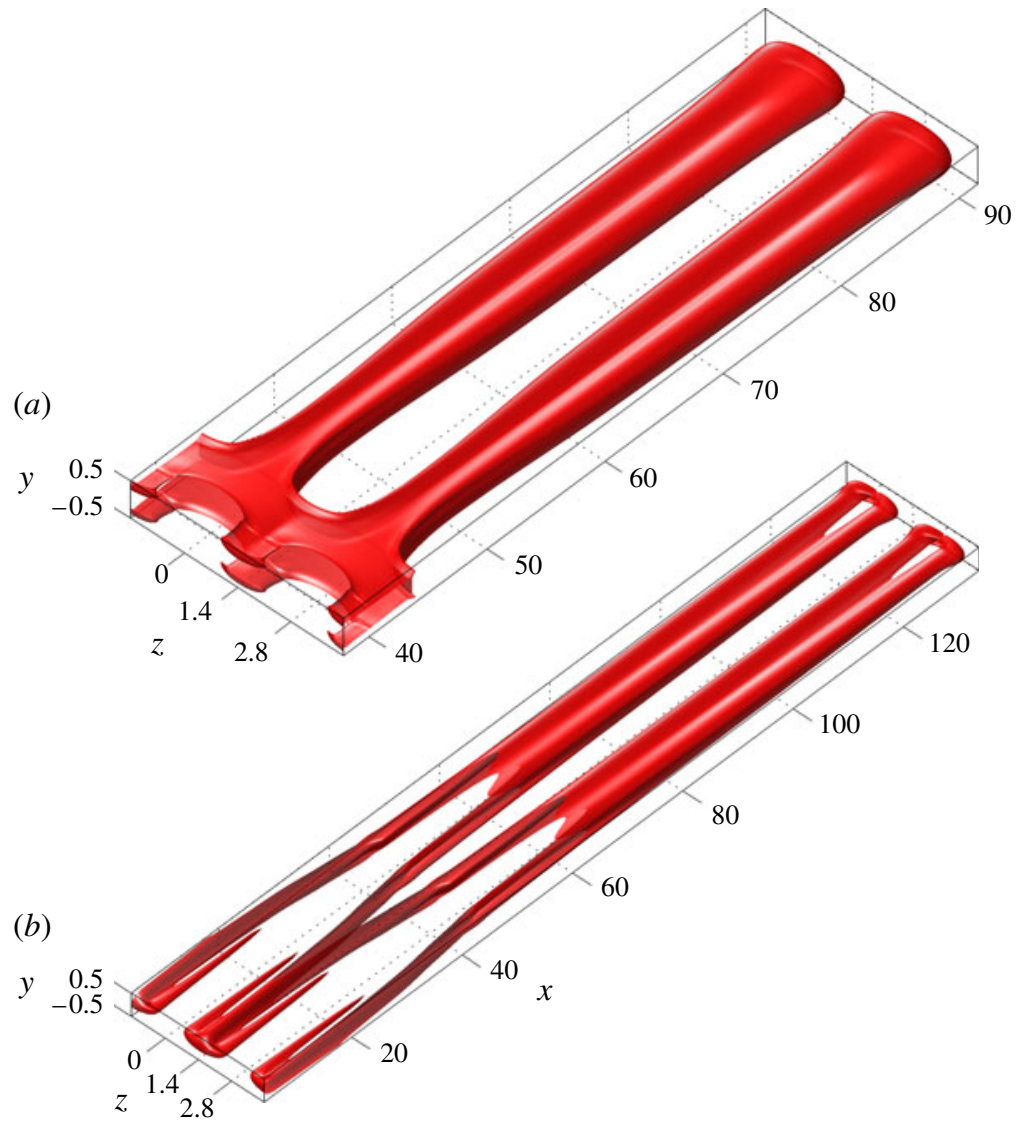

Figure 12. (Colour online) Perspective view of the three-dimensional evolution for $M=$ 3, $F=20, P e=1000$ and $R e^{*}=1$, at times $(a) t=20$ and $(b) t=38$. For clarity, two spanwise wavelengths are shown. The upward-propagating finger of light fluid undergoes an inner splitting event, similar to what happens in the neutrally buoyant case. A second, dense front moves downward in the $-x$ direction and eventually forms an anchor-like shape. The concentration value of $c=0.35$ was chosen as it allows us to visualize both of these instabilities in $(b)$.

inside the quadrupole within each $x=$ constant cross-section, and then determine its global maximum $\Omega_{x, \max }$ over all $x$-locations. Figure 15 shows the temporal evolution of $\Omega_{x, \max }$ for $M=3, P e=1000, R e^{*}=1$ and various gravity numbers $F$. Higher levels of gravitational forces are seen to result in a more rapid growth of the quadrupole's strength during the initial tip acceleration phase. After reaching a peak, $\Omega_{x, \max }$ decreases again. During the late stages of the finger's evolution, larger $F$-values are still associated with stronger quadrupoles, although the dependence is relatively mild.

The finding of stronger inner quadrupoles for larger $F$-values suggests that inner splitting will occur more rapidly at larger $F$-values. However, figure 16 shows the exact opposite behaviour, i.e. the same $c$-contours split later for larger $F$. This indicates that the strength of the inner quadrupole is not solely responsible for driving the inner splitting. Rather, with increasing density stratification the strength of the opposite-signed, outer quadrupole vortex also increases, which serves to delay the inner splitting. For large $F$-values, this secondary quadrupole is significantly stronger 

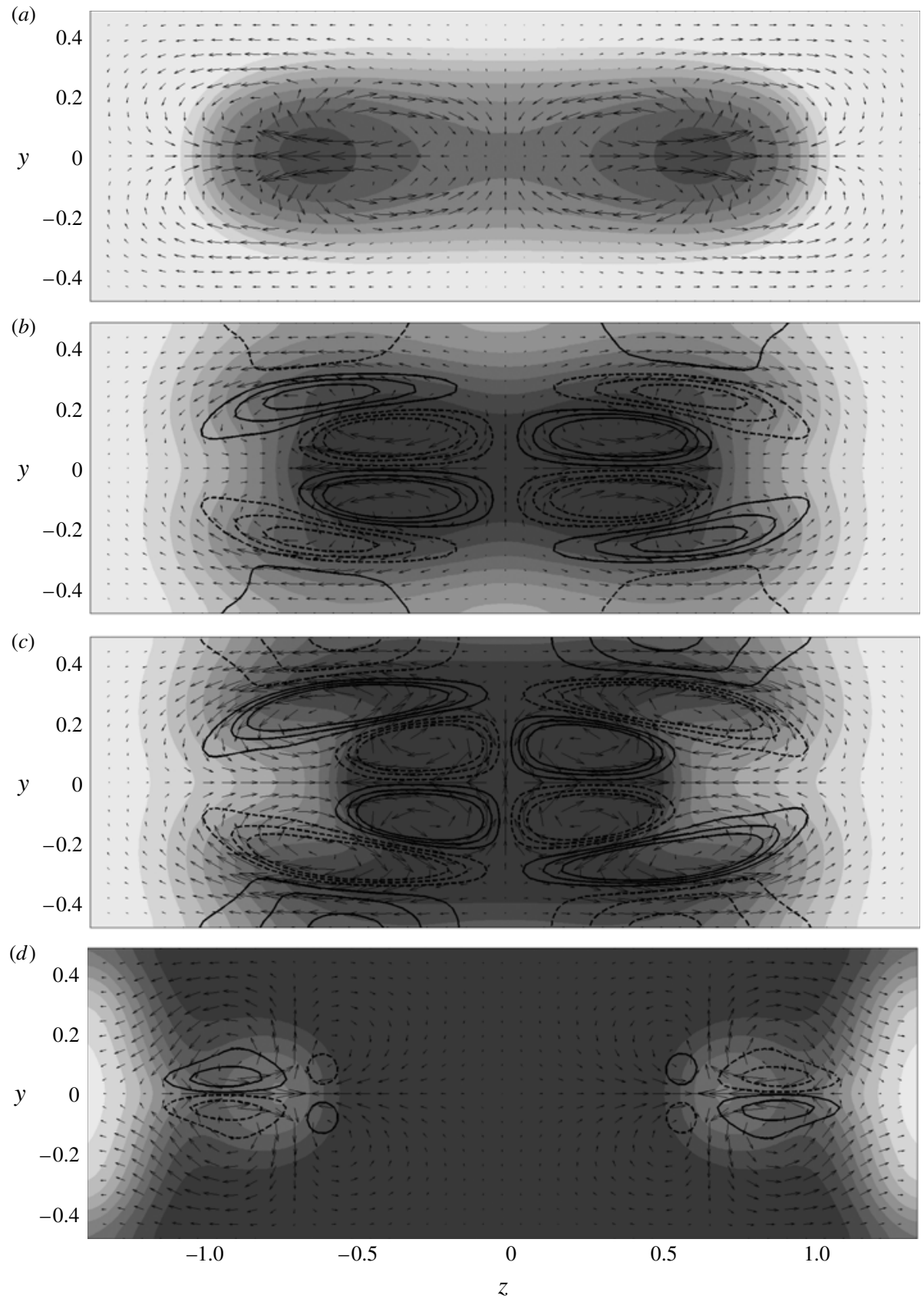

Figure 13. Cross-sections at $(a) x=129,(b) x=80$, (c) $x=55$ and $(d) x=20$ for the flow shown in figure $12(b)$. Grey shading indicates the concentration field, with darker regions corresponding to lighter, less-viscous injected fluid. The arrows show the $(y, z)$ velocity field, while the solid (dashed) lines in $(b)-(d)$ represent contours of negative (positive) streamwise vorticity. 

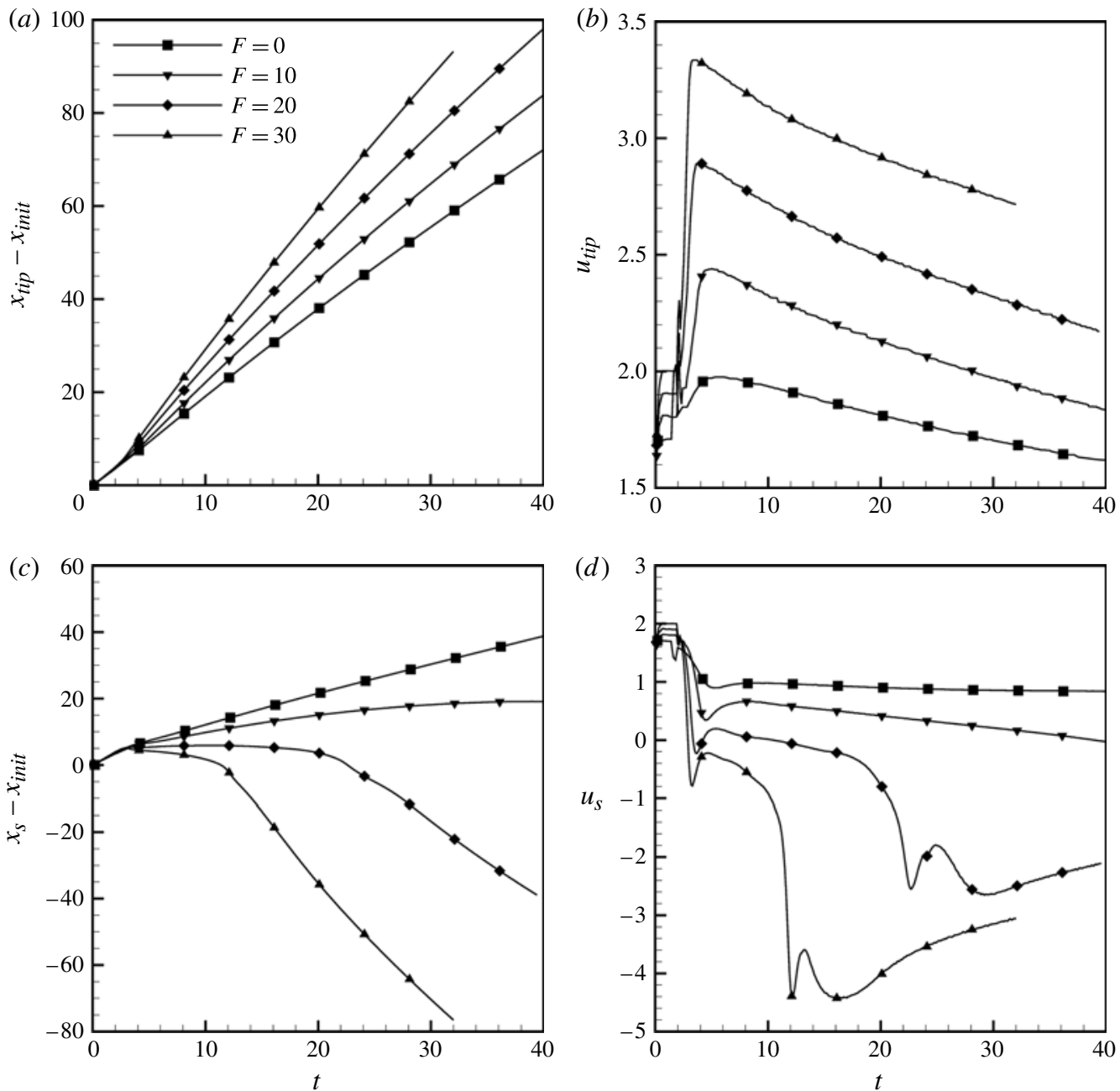

FIGURE 14. Tip locations and velocities of the upward- and downward-propagating fronts for $M=3, P e=1000, R e^{*}=1$ and various values of $F$. (a) Temporal evolution of the upward-propagating finger tip position $x_{\text {tip }}$ relative to the initial interface position $x_{\text {init }}$. (b) Evolution of the upward tip velocity $u_{t i p}$, defined as $u_{t i p}=\mathrm{d} x_{t i p} / \mathrm{d} t$ : generally, a stronger unstable density stratification causes the finger to advance more rapidly; after reaching an initial peak, the tip velocity gradually decreases over time. (c) Location of the trailing front $x_{s}$ relative to the initial interface position $x_{\text {init }}$ as a function of time. $(d)$ Evolution of the trailing front velocity $u_{s}$, defined as $u_{s}=\mathrm{d} x_{s} / \mathrm{d} t$ : for weak unstable density stratification the trailing front still propagates upward; however, as $F$ increases, it reverses direction and propagates downward, driven by the gravitational instability.

than in the neutrally buoyant case, which is also reflected by the cross-sectional finger shapes seen in figure 13(b,c). Another contributing factor to delayed splitting is that even though the finger moves faster for larger $F$, it is also thicker across the gap, and so it takes longer for the streamwise vorticity to split the finger.

Comparing the time-evolution of the inner splitting to the temporal appearance of the pinch-off in the two-dimensional case (cf. figure $5 a$ ) reveals some similarity in the 


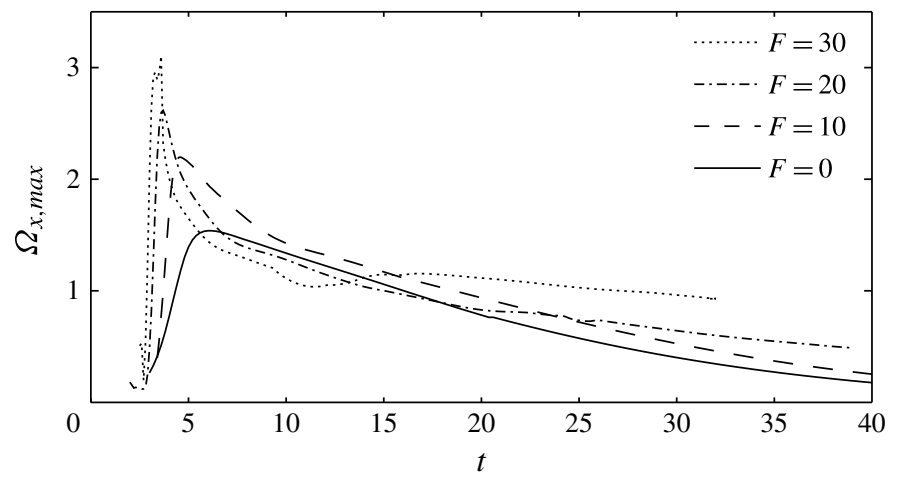

FIGURE 15. Temporal evolution of the inner quadrupole's maximum streamwise vorticity $\Omega_{x, \max }$, for $M=3, P e=1000, R e^{*}=1$ and various values of $F$. Higher levels of density stratification result in stronger quadrupoles.

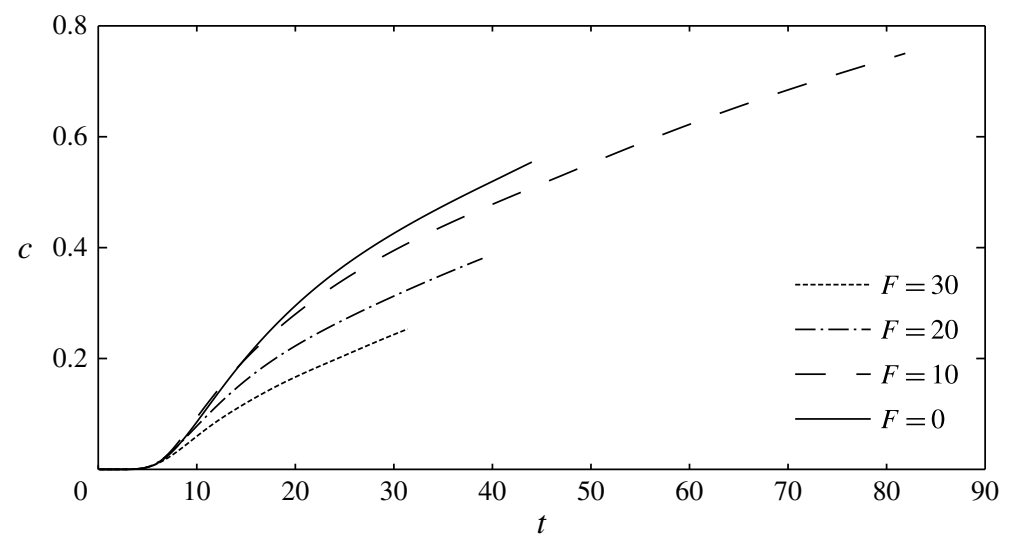

FIGURE 16. Evolution of inner splitting for continuous concentration levels as a function of time $t$ for $M=3, P e=1000$ and increasing gravity numbers $F=0,10,20$ and 30 . Stronger density stratification results in later splitting.

sense that both the three-dimensional inner splitting and the two-dimensional pinch-off are somewhat delayed for increasing $F$-values. However, the influence of $F$ on the splitting mechanism is significantly stronger, while the pinch-off is mostly dominated by diffusive effects.

We now focus on the evolution of the anchor-like structure at the trailing front through gravitational instability. Figure 17 shows perspective views of the $c=0.5$ concentration contour for $M=3, F=20, P e=1000$ and $R e^{*}=1$ at times $t=23.5,25$, 27 and 30.5. For this purpose, both flow and concentration data have been mirrored at the side symmetry plane $z=-L_{z} / 2$. The trailing front's deformation, leading to the emergence of the distinctive anchor shape, represents a two-stage process. Early on, the interface shape resembles the typical mushroom structure familiar from RayleighTaylor instabilities; cf. figure 17( $a, b)$ and the results in Sharp (1984), Fernandez et al. (2002), Graf, Meiburg \& Härtel (2002) and Martin, Rakotomalala \& Salin (2002). Subsequently, this mushroom is stretched longitudinally as it sinks towards smaller $x$-locations. During the late stages, a pair of holes form in the anchor, reflecting the 
(a)
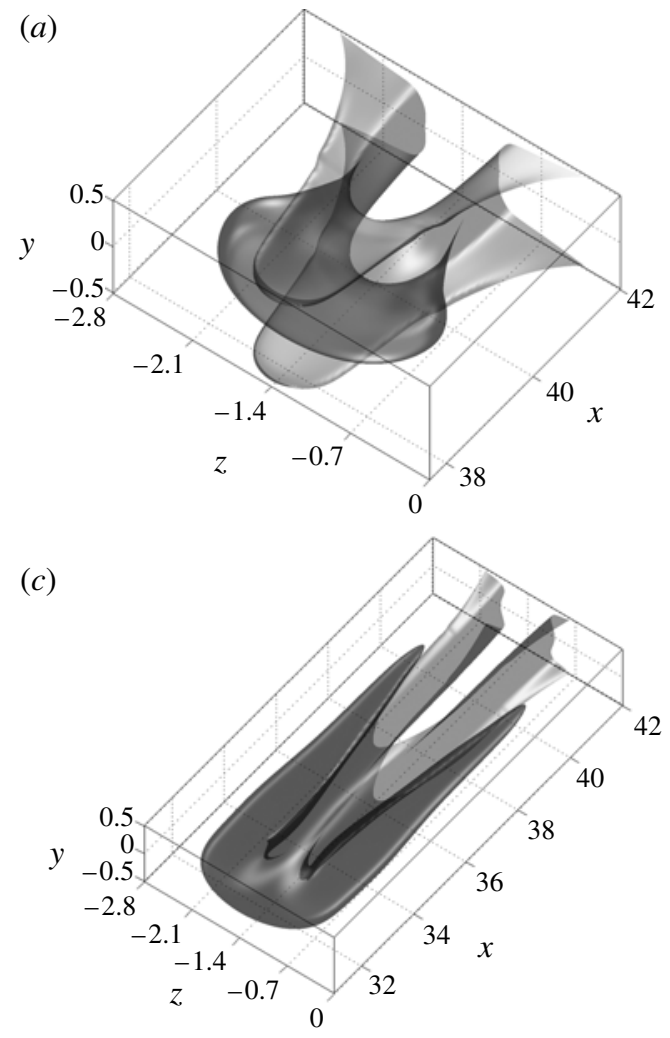

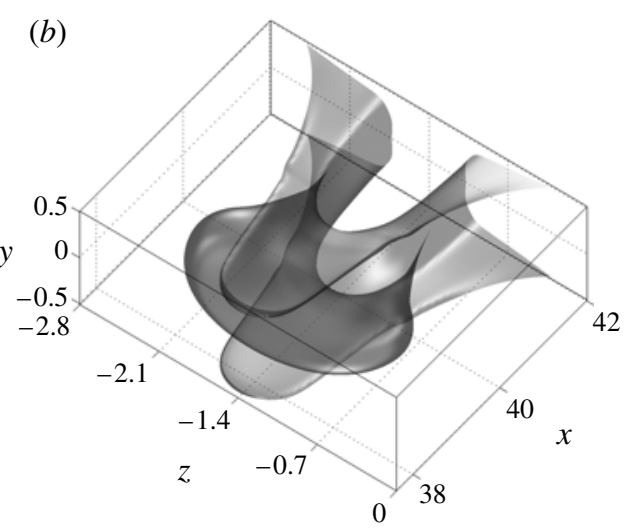

(d)

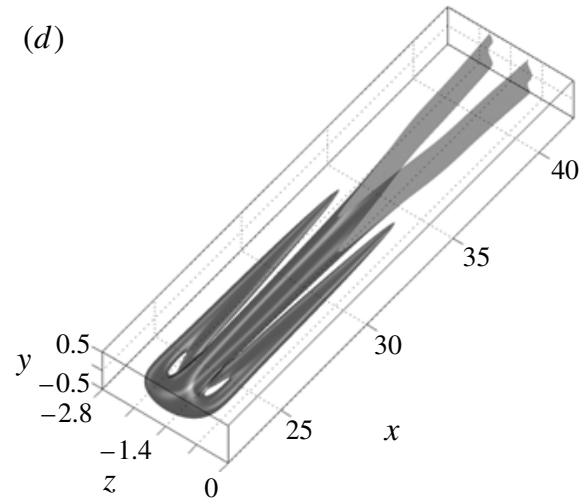

FIgURE 17. Three-dimensional profiles of the concentration isosurface $c=0.5$ for $M=3$, $F=20$ and $P e=1000$, at times $(a) t=23.5,(b) t=25,(c) t=27$ and $(d) t=30.5$.

action of the streamwise vorticity quadrupole described earlier. This process can be observed in more detail in the succession of two-dimensional cross-cuts shown in figures 18 and 19 . We remark that the pronounced unsteadiness in the trailing front velocity between $t \approx 23$ and $t \approx 29$ in figure $14(d)$ is associated with the emergence of the mushroom shape and its subsequent stretching into the anchor. The anchor then approaches a quasisteady shape and continues to propagate further downward for $t \gtrsim 29$.

Figure 18 shows two-dimensional $c=0.5$ concentration contours of the flow in figure 17, in the centre-plane $y=0$ of the Hele-Shaw cell. The formation of the mushroom structure and its subsequent stretching into the anchor-like shape are evident. In figure $18(d)$ we see the influence of the vorticity quadrupole on the anchor formation in the presence of two closed contours located downstream of the downward-moving front, as well as in the elongated gap between the anchor stem and its flukes; cf. figure $13(d)$. Figure 18(e) indicates that the $c=0.5$ contour goes through a reconnection process before defining the final shape of the anchor. Figure 19 displays corresponding contours in the anchor's cross-gap symmetry plane $z=-L_{z} / 2$. The denser and more-viscous fluid initially travels upwards. It subsequently reverses direction and begins to sink downward in the $-x$ direction. During this transition, shown in figure $19(b)$, we have two displacement fronts travelling close to the walls, instead of a single front travelling along the cell centre. 


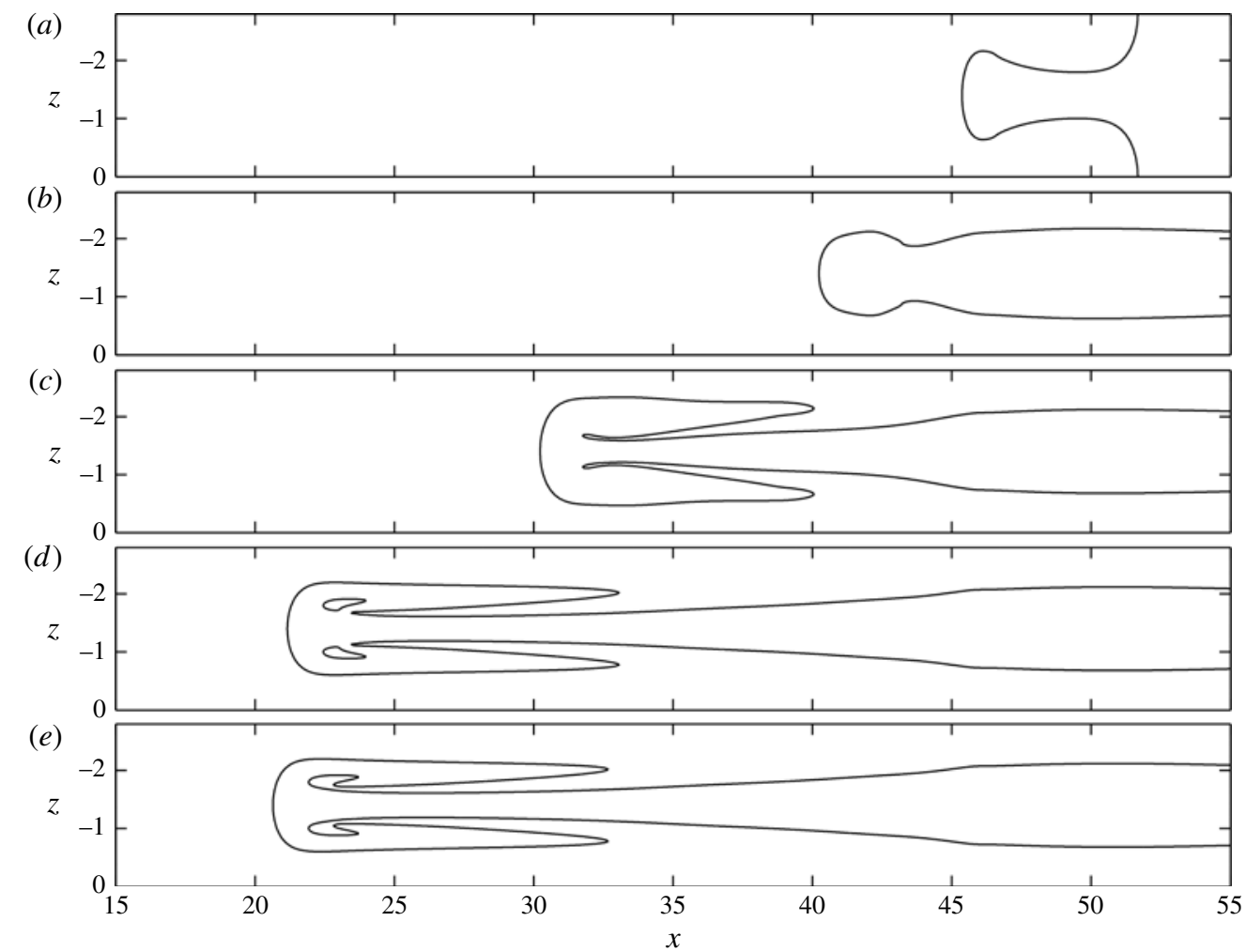

FIgURE 18. Two-dimensional $c=0.5$ concentration contours in the central symmetry plane $y=0$, for the same parameters as in figure 17 at times $t=5,22.5,27.3,30.8$ and 31. The initial formation of the mushroom structure, and its subsequent stretching into the anchor shape, can be identified clearly. A streamwise vorticity quadrupole injects less-viscous fluid between the anchor stem and its flukes, thereby generating the elongated gap visible in the figure.

One-dimensional concentration profiles $c_{a v g}(x)$ are obtained by averaging across the entire $(y, z)$ cross-section. Figure 20(a) displays such cross-sectional averages for $M=$ 3, $F=30, P e=1000$ and $R e^{*}=1$, at equidistant times. The upward propagation of a frontal shock with a velocity greater than the maximum Poiseuille flow velocity of 1.5 is evident, along with a downward-propagating shock reflecting the presence of the anchor.

Figure 20(b) compares the cross-sectionally averaged concentration profiles for gravity numbers $F=0,10,20$ and 30 at the fixed time $t=30$. While the upward-propagating shock exists for all $F$-values, a downward-moving shock is observed only for $F=20$ and 30. At later times the downward-moving shock is observed for $F=10$ as well.

\section{Tip splitting versus inner splitting}

Let us increase the wavelength of the imposed perturbation to $1.5 \lambda_{m}$ and increase the spanwise domain size accordingly. In this situation, the viscous finger becomes susceptible to developing a tip-splitting instability as well. This phenomenon is better understood than the inner splitting instability and has been observed in both miscible 

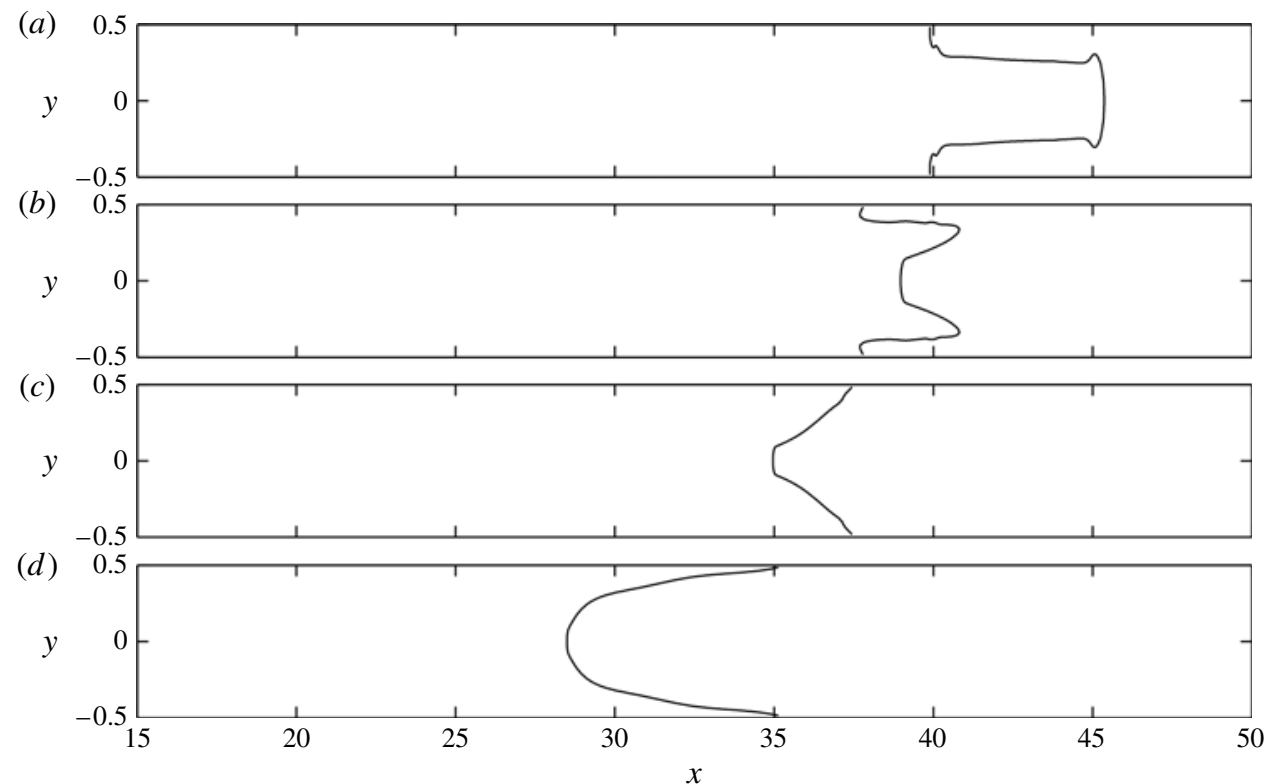

FIGURE 19. Two-dimensional $c=0.5$ concentration contours in the side symmetry plane $z=-L_{z} / 2$, for the same parameters as in figure 17 at times $t=5,23,25$ and 28. After initially propagating upward in the $+x$ direction, the dense fluid reverses direction and sinks downward, driven by the unstable density stratification.

and immiscible displacements; see Kopf-Sill \& Homsy (1988), Tan \& Homsy (1988), Lajeunesse \& Couder (2000) and Dimakopoulos \& Tsamopoulos (2003). Figure 21 displays the front of the $c=0.3$ concentration isosurface for $(M, P e, F)=(3,1000,20)$ at times $t=26.3,27.9,28.7,29.5$ and 30.3. The time-evolution of this concentration value shows the widening and flattening of tip followed by the emergence of a small dimple at the foremost point of the tip, which subsequently grows and triggers the splitting of the tip. Behind the front, the top and bottom surfaces bend inwards and meet at the midgap plane, so that diffusion can result in the inner splitting instability. The evolution of these two instabilities can be seen in panels $(a)-(e)$. At a later time, the small 'bridge' linking the two instabilities diffuses away, so that the finger is split into two longitudinal parts. For comparison, figure $21(f)$ shows the $c=0.5$ concentration isosurface at the same time as in panel $(e)$. The inner splitting of the $c=0.3$ surface occurs at about the same time for the fastest-growing mode $\lambda_{m}$ and for the longer-wavelength mode with $1.5 \lambda_{m}$. As can be seen in figure 16, the inner splitting occurs later for increasing $c$-values. Because this instability is diffusively dominated, a considerable amount of time can pass between the inner splitting of surfaces of successively larger concentration values. The tip splitting, on the other hand, is convectively dominated and occurs concurrently for all $c$-values.

\section{Summary and conclusions}

We have investigated gravitationally and viscously unstable miscible displacements in vertical Hele-Shaw cells via three-dimensional Navier-Stokes simulations. For the two-dimensional base flows, these simulations show that the propagation velocity of the displacement front increases with the unfavourable viscosity contrast and 

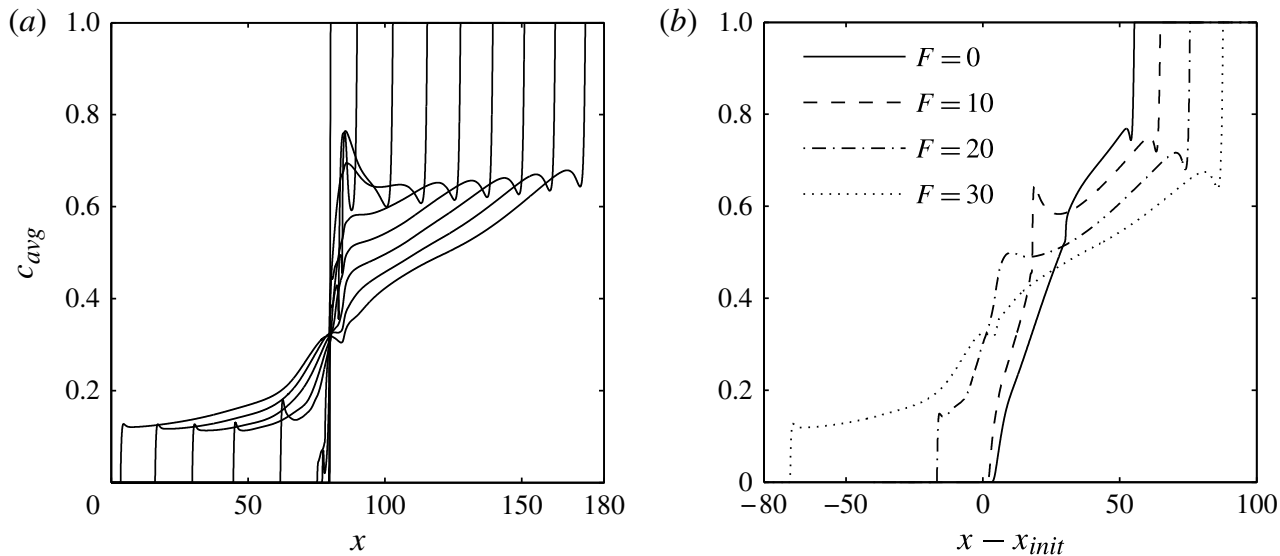

FIGURE 20. Cross-sectionally averaged concentration profiles for $M=3, P e=1000$ and $R e^{*}=1$ : (a) growth of both the viscous finger and the upstream Rayleigh-Taylor instability for $F=30$ at $t=0,4,8, \ldots, 32 ;(b)$ averaged concentration profiles at a fixed time $t=30$ for increasing gravity numbers $F=0,10,20$ and 30 .

the destabilizing density difference. We find that displacement fronts moving faster than the maximum velocity of the Poiseuille flow far downstream exhibit a single stagnation point in a moving reference frame, consistent with earlier observations by Taylor (1961) and Petitjeans \& Maxworthy (1996) for corresponding capillary tube flows. For stabilizing density differences, on the other hand, the front can move more slowly than the Poiseuille flow, and more complex streamline patterns emerge, which allow the formation of a spike at the tip of the front, consistent with earlier observations by Lajeunesse et al. (1999) and Goyal et al. (2007). The simulations furthermore demonstrate a two-dimensional pinch-off some distance behind the displacement front, which is largely governed by diffusive rather than gravitational effects.

Three-dimensional simulations of viscously and gravitationally unstable vertical displacements show a strong vorticity quadrupole along the length of the finger. This quadrupole is qualitatively similar to the one observed by Oliveira \& Meiburg (2011) for neutrally buoyant flows, and just as for neutrally buoyant displacements it also leads to an inner splitting instability of fingers in vertical displacements. We find that the quadrupole's strength increases for larger destabilizing density differences. Nevertheless, the inner splitting is delayed for increasing density differences, due to the presence of a secondary, outer quadrupole which counteracts the inner one. For large unstable density differences, we furthermore observe the formation of a secondary, downward-propagating front that is also characterized by inner and outer vorticity quadrupoles. The flow induced by these quadrupoles results in an anchor-like shape of the downward-propagating front.

By increasing the initial spanwise perturbation wavelength, we can trigger the formation of the well-known tip-splitting instability, as well as its concurrent growth with the novel inner splitting instability. While each of these two instabilities causes a subharmonic bifurcation of the overall finger structure, they primarily affect different segments of this finger, so they are not in direct competition with each other and can appear simultaneously.

In light of the novel two-dimensional findings recently reported by Taghavi, Alba \& Frigaard (2012a) and Taghavi et al. (2012b) for miscible displacements in inclined 
(a)

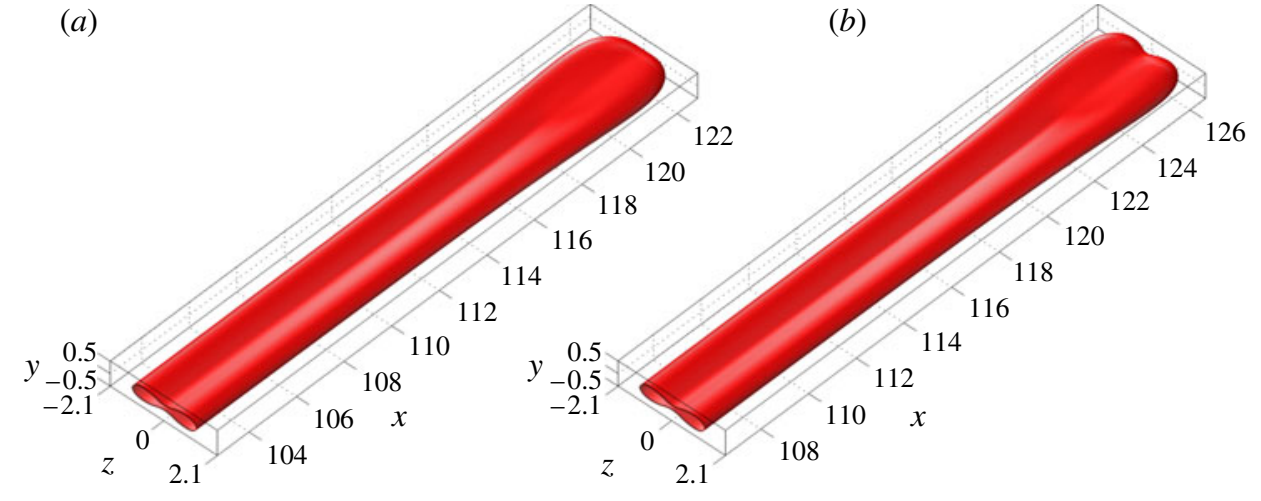

(c)

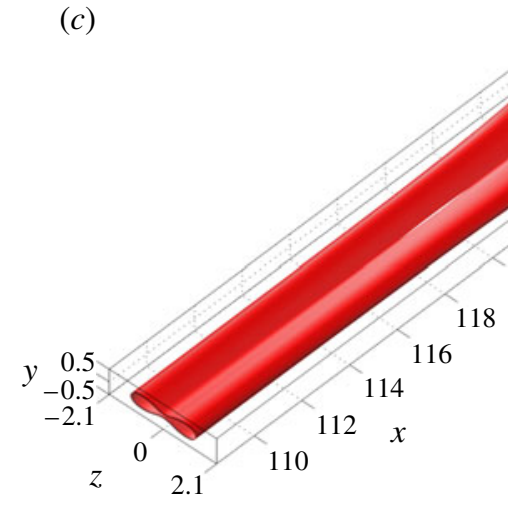

$(e)$

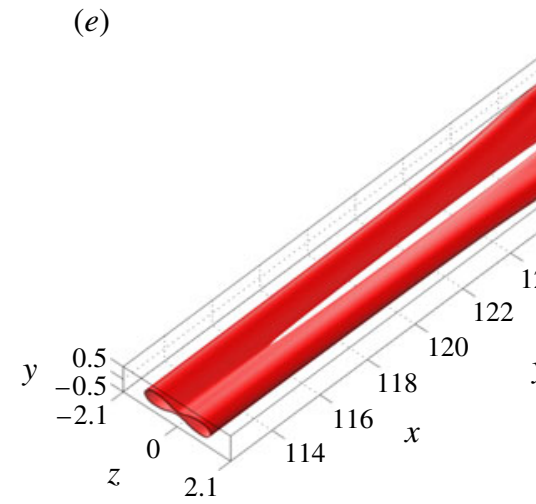

$(d)$

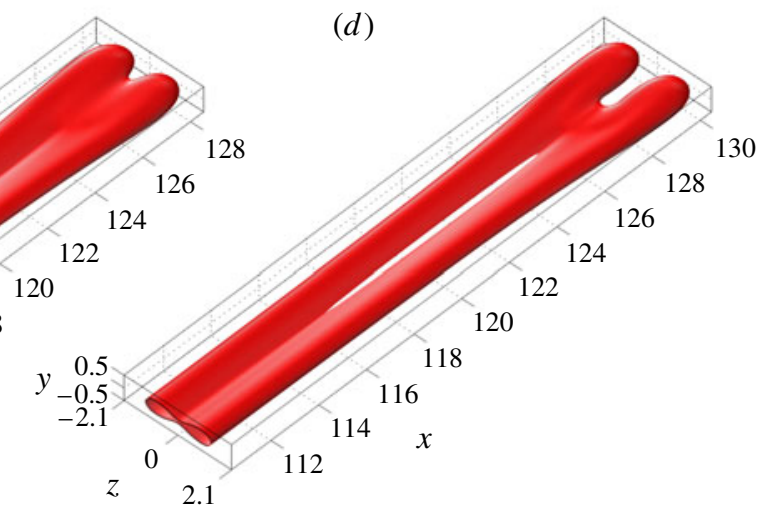

$(f)$
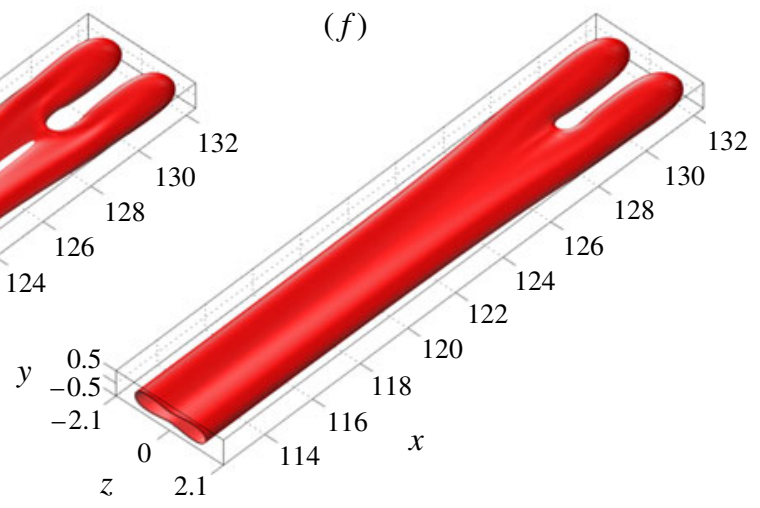

Figure 21. (Colour online) The $c=0.3$ isosurface for $(M, P e, F)=(3,1000,20)$ at times (a) $t=26.3$, (b) $t=27.9$, (c) $t=28.7,(d) t=29.5$ and $(e) t=30.3$. The spanwise domain width and the perturbation wavelength are $1.5 \lambda_{m}$. The temporal evolution shows the concurrent emergence of tip splitting and inner splitting. $(f)$ Concentration isosurface $c=0.5$ at time $t=30.3$, for comparison.

channels, it will be interesting to explore how the three-dimensional effects observed here and in John et al. (2013) for purely vertical and horizontal displacements interact under the more general conditions of inclined channels. 


\section{Acknowledgements}

E.M. acknowledges financial support through NSF grant CBET-0651498 and DOE grant DE-FG02-08ER15991, and from the Petroleum Research Fund, administered by the American Chemical Society, under grant 45175-AC9. R.M.O. would like to thank the CAPES Foundation of Brazil and the Fulbright programme for financial support through grants BEX 2615/06-1 and IIE 15073695. High-performance computing support was obtained through the California NanoSystems Institute under NSF grant CHE-0321368, through Centro Nacional de Supercomputação (CESUP) located at UFRGS, Brazil, and through the Community Surface Dynamics Modeling System (CSDMS) at the University of Colorado, Boulder. We would like to thank CSDMS director Professor James Syvitski and the technical staff at CSDMS for their support.

\section{REFERENCES}

Alba, K., Taghavi, S. M. \& FrigaArd, I. A. 2012 Miscible density-stable displacement flows in inclined tube. Phys. Fluids 24, 123102.

Chen, C.-Y. \& Meiburg, E. 1996 Miscible displacements in a capillary tube. Part 2. Numerical simulations. J. Fluid Mech. 326, 57-90.

Chen, C.-Y. \& Meiburg, E. 1998 Miscible porous media displacements in the quarter five-spot configuration. Part 2. Effect of heterogeneities. J. Fluid Mech. 371, 269-299.

D'Errico, G., Ortona, O., Capuano, F. \& Vitagliano, V. 2004 Diffusion coefficients for the binary system glycerol + water at $25^{\circ} \mathrm{C}$ : a velocity correlation study. J. Chem. Engng Data 49, $1665-1670$.

Dimakopoulos, Y. \& Tsamopoulos, J. 2003 Transient displacement of a Newtonian fluid by air in straight or suddenly constricted tubes. Phys. Fluids 15, 1973-1991.

Fairbrother, F. \& Stubbs, A. E. 1935 Studies in electro-endosmosis. Part VI. The 'bubble-tube' method of measurement. J. Chem. Soc. 1, 527-529.

Fernandez, J., Kurowski, P., Petitjeans, P. \& Meiburg, E. 2002 Density-driven unstable flows of miscible fluids in a Hele-Shaw cell. J. Fluid Mech. 451, 239-260.

Goyal, N. \& Meiburg, E. 2006 Miscible displacements in Hele-Shaw cells: two-dimensional base states and their linear stability. J. Fluid Mech. 558, 329-355.

Goyal, N., Pichler, H. \& Meiburg, E. 2007 Variable-density miscible displacements in a vertical Hele-Shaw cell: linear stability. J. Fluid Mech. 584, 357-372.

Graf, F., Meiburg, E. \& Härtel, C. 2002 Density-driven instabilities of miscible fluids in a Hele-Shaw cell: linear stability analysis of the three-dimensional Stokes equations. J. Fluid Mech. 451, 261-282.

Homsy, G. M. 1987 Viscous fingering in porous media. Annu. Rev. Fluid Mech. 19, 271-311.

John, M. O., Oliveira, R. M., Heussler, F. H. C. \& Meiburg, E. 2013 Variable density and viscosity, miscible displacements in horizontal Hele-Shaw cells. Part 2. Nonlinear simulations. J. Fluid Mech. 721, 295-323.

KIM, J. \& MoIN, P. 1985 Application of a fractional-step method to incompressible Navier-Stokes equations. J. Comput. Phys. 59, 308-323.

Kopf-Sill, A. R. \& Homsy, G. M. 1988 Nonlinear unstable viscous fingers in Hele-Shaw flows. I. Experiments. Phys. Fluids 31, 242-249.

Kuang, J., Petitjeans, P. \& Maxworthy, T. 2004 Velocity fields and streamline patterns of miscible displacements in cylindrical tubes. Exp. Fluids 37, 301-308.

LAJeunesse, E. \& Couder, Y. 2000 On the tip-splitting instability of viscous fingers. J. Fluid Mech. 419, 125-149.

Lajeunesse, E., Martin, J., Rakotomalala, N. \& Salin, D. 1997 3D instabilities of miscible displacements in a Hele-Shaw cell. Phys. Rev. Lett. 79 (26), 5254-5257.

Lajeunesse, E., Martin, J., Rakotomalala, N. \& Salin, D. 2001 The threshold of the instability in miscible displacement in a Hele-Shaw cell at high rates. Phys. Fluids 13 (3), 799-801. 
Lajeunesse, E., Martin, J., Rakotomalala, N., Salin, D. \& Yortsos, Y. C. 1999 Miscible displacement in a Hele-Shaw cell at high rates. J. Fluid Mech. 398, 229-319.

Martin, J., Rakotomalala, N. \& Salin, D. 2002 Gravitational instability of miscible fluids in a Hele-Shaw cell. Phys. Fluids 14 (2), 902-905.

MaXworthy, T. 1987 The nonlinear growth of a gravitationally unstable interface in a Hele-Shaw cell. J. Fluid Mech. 177, 207-232.

Oliveira, R. M. \& Meiburg, E. 2011 Miscible displacements in Hele-Shaw cells: three-dimensional Navier-Stokes simulations. J. Fluid Mech. 687, 431-460.

PARK, C. W. \& Homsy, G. M. 1984 Two phase displacement in Hele-Shaw cells: theory. J. Fluid Mech. 139, 291-308.

Petitjeans, P. \& Maxworthy, T. 1996 Miscible displacements in capillary tubes. Part 1. Experiments. J. Fluid Mech. 326, 37-56.

RAI, M. M. \& MoIN, P. 1991 Direct simulations of turbulent flow using finite-difference schemes. J. Comput. Phys. 96 (1), 15-53.

Rakotomalala, N., Salin, D. \& Watzky, P. 1997 Miscible displacement between two parallel plates: BGK lattice gas simulations. J. Fluid Mech. 338, 277-297.

Rashidnia, N. \& Balasubramaniam, R. 2004 Measurement of the mass diffusivity of miscible liquids as a function of concentration using a common path shearing interferometer. Exp. Fluids 36, 619-626.

Ruith, M. \& Meiburg, E. 2000 Miscible rectilinear displacements with gravity override. Part 1. Homogeneous porous medium. J. Fluid Mech. 420, 225-257.

Sharp, D. H. 1984 An overview of Rayleigh-Taylor instability. Physica D 12, 3-18.

SoARES, E. J.\& THOMPson, R. L. 2009 Flow regimes for the immiscible liquid-liquid displacement in capillary tubes with complete wetting of the displaced liquid. J. Fluid Mech. 641, 63-84.

Taghavi, S. M., Alba, K. \& FrigaArd, I. A. 2012a Buoyant miscible displacement flows at moderate viscosity ratios and low Atwood numbers in near-horizontal ducts. Chem. Engng Sci. 69, 404-418.

Taghavi, S. M., Alba, K., Seon, T., Wielage-Burchard, K., Martinez, D. M. \& Frigaard, I. A. $2012 b$ Miscible displacement flows in near-horizontal ducts at low Atwood number.

J. Fluid Mech. 696, 175-214.

Talon, L., Goyal, N. \& Meiburg, E. 2013 Variable density and viscosity, miscible displacements in horizontal Hele-Shaw cells. Part 1. Linear stability analysis. J. Fluid Mech. 721, 268-294.

TAN, C. T. \& Homsy, G. M. 1988 Simulation of nonlinear viscous fingering in miscible displacement. Phys. Fluids 31 (6), 1330-1338.

TAYLOR, G. I. 1961 Deposition of a viscous fluid on the wall of a tube. J. Fluid Mech. 10, 161-165. 